\title{
A surrogate based multistage-multilevel optimization procedure for multidisciplinary design optimization
}

\author{
Wen Yao • Xiaoqian Chen • Qi Ouyang • \\ Michel van Tooren
}

Received: 19 May 2011 / Revised: 9 August 2011 / Accepted: 30 August 2011 / Published online: 27 September 2011

(C) Springer-Verlag 2011

\begin{abstract}
Optimization procedure is one of the key techniques to address the computational and organizational complexities of multidisciplinary design optimization (MDO). Motivated by the idea of synthetically exploiting the advantage of multiple existing optimization procedures and meanwhile complying with the general process of satellite system design optimization in conceptual design phase, a multistage-multilevel MDO procedure is proposed in this paper by integrating multiple-discipline-feasible (MDF) and concurrent subspace optimization (CSSO), termed as MDFCSSO. In the first stage, the approximation surrogates of high-fidelity disciplinary models are built by disciplinary specialists independently, based on which the single level optimization procedure MDF is used to quickly identify the promising region and roughly locate the optimum of the MDO problem. In the second stage, the disciplinary specialists are employed to further investigate and improve the baseline design obtained in the first stage with highfidelity disciplinary models. CSSO is used to organize the concurrent disciplinary optimization and system coordination so as to allow disciplinary autonomy. To enhance the reliability and robustness of the design under uncertainties, the probabilistic version of MDF-CSSO (PMDF-CSSO) is developed to solve uncertainty-based optimization problems. The effectiveness of the proposed methods is verified
\end{abstract}

\footnotetext{
W. Yao $\cdot$ X. Chen $\cdot$ Q. Ouyang

College of Aerospace and Materials Engineering,

National University of Defense Technology,

Changsha 410073, China

W. Yao $(\varangle) \cdot$ M. van Tooren

Faculty of Aerospace Engineering,

Delft University of Technology,

Delft 2629HS, The Netherlands

e-mail: wendy0782@126.com
}

with one MDO benchmark test and one practical satellite conceptual design optimization problem, followed by conclusion remarks and future research prospects.

Keywords Multidisciplinary design optimization (MDO) . Optimization procedure - Multiple-discipline-feasible (MDF) · Concurrent subspace optimization (CSSO) . Surrogate model

\section{Introduction}

To address the optimization problems of complex systems involving multiple close coupled disciplines, the methodology multidisciplinary design optimization (MDO) is widely studied and applied in both academia and industry. MDO can enhance system design by exploiting synergies among different disciplines. However, there are two major challenges in applying MDO, namely computational and organizational complexities. To address these two challenges, one of the research focuses in MDO has been on optimization procedure (Sobieszczanski-Sobieski and Haftka 1997). Optimization procedure organizes MDO elements, e.g. sensitivity analysis, design space search, system or disciplinary analysis, etc., into executable sequences. Generally optimization procedures can be categorized into two types: single-level and multi-level approaches. Singlelevel approaches employ a system optimizer for the whole problem, which is straightforward to understand and easy to implement. Typical single-level approaches include multiple-discipline-feasible (MDF), individual-disciplinefeasible (IDF), all-at-once (AAO), and simultaneous analysis and design (SAND). Multi-level approaches utilize decomposition strategies to allow disciplinary autonomy 
in design and optimization, meanwhile manage interdisciplinary consistence by system coordination. Typical multi-level approaches include concurrent subspace optimization (CSSO), collaborative optimization (CO), bi-level integrated system synthesis (BLISS), and analytical target cascading (ATC). These procedures are investigated and compared with benchmark tests (Balling and SobieszcznskiSobieski 1996; Balling and Wilkinson 1997; Alexandrov and Kodiyalam 1998; Chen et al. 2002; Yi et al. 2008; 2010), and the results show that none of them is universally good. Generally single-level approaches are more robust in terms of convergence and computational efficiency, whereas multi-level approaches are greatly influenced by the characteristics of the specific problems under study (e.g. the degree of interdisciplinary interaction) and the implementation details to realize decomposition and coordination, e.g. approximation techniques, system sensitivity analysis, etc. For example, $\mathrm{CO}$ may encounter convergence problem if the formulation is degenerate (DeMiguel and Murray 2006), and BLISS may entail lots of iterative cycles to converge if approximation models are inaccurate or initial bounds on design variables are not properly defined (Zhao and Cui 2011). The standard CSSO is also shown to be computationally inefficient as too many function calls are needed to converge (Yi et al. 2008), but the use of approximation surrogates can bring a 1-2 order of magnitude reduction in the number of system analyses compared to AAO (Sellar and Batill 1996; Sellar et al. 1996b; Simpson et al. 2004). Thus the performances of MDO procedures are problem and implementation dependent, and the selection of a proper optimization procedure for a specific problem is more or less in an ad hoc manner.

Since each optimization procedure has pros and cons, there is possibility to synthetically utilize different optimization procedures to solve MDO problems, so as to enhance effectiveness and efficiency by exploiting advantages and circumventing drawbacks of different approaches. This idea is similar to the multi-method collaborative optimization (MCO) approach which combines different search algorithms to enhance global optimization capability (Luo 2003). Zhao and Cui developed a bi-Level integrated system collaborative optimization (BLISCO) procedure by integrating the collaborative thought of $\mathrm{CO}$ and the main characteristic of BLISS-2000, which proved to be more reliable than $\mathrm{CO}$ in terms of convergence and more efficient than BLISS with less iterative cycles to converge (Zhao and Cui 2011). Inspired by this thought, a MDO procedure is proposed by combining MDF and CSSO in this paper. The motivation for this combination is to comply with the realistic satellite design and optimization process, which includes the following two points.

First, in conceptual design phase, after the objectives and constraints of satellite system design are defined, candidate schemes can be generated and evaluated. As no a priori knowledge is available about the potentially good solution, a large set of candidates should be investigated, which can be realized by optimization to identify the optimal solution. In this stage, mainly the system level specialists are involved for decision making, and low-fidelity models are usually used to accelerate the process to obtain a feasible and preferable design as the baseline for further investigation. This fast system level optimization can be realized by surrogate based MDF. The reason for selection of MDF among other procedures is that MDF directly solve the original optimization problem without mathematical reformulation, which is easy to implement and stable in convergence and optimization effectiveness. Besides, the major obstacle of MDF is the prohibitive computation caused by multidisciplinary analysis (MDA) which is repeatedly called during optimization, as MDA of coupled disciplines entails iterations of disciplinary analyses to reach a consistent result for a design. This obstacle can be eliminated as surrogates are used. Hence surrogate based MDF is viable for the system level optimization to quickly obtain the preferable baseline.

Second, based on the baseline, disciplinary specialists are employed to study and improve the design with high-fidelity tools under system level management. The disciplinary specialists with their own analysis tools are usually geographically dispersed and operate with relative independence. Thus autonomy for disciplinary optimization is desired. In this stage, decomposition based procedures are needed to allow disciplinary autonomy, and CSSO is preferred due to its resemblance to the real-world disciplinary organization without significant changes in the objective and constraint formulations.

As MDF and CSSO are combined to solve the aforementioned two stage design optimization problem involving the system and disciplinary levels, this MDF-CSSO procedure is a multistage-multilevel MDO procedure.

In realistic engineering, there exist uncertainties which should be considered to address issues of reliability and robustness. Uncertainties can be categorized into two types: aleatory and epistemic. Aleatory uncertainty describes the inherent variation of the physical system or environment under study. Epistemic uncertainty is a potential inaccuracy that is due to a lack of knowledge (Hoffman and Hammonds 1994; Helton and Burmaster 1996). The aleatory uncertainties are generally modeled as random variables with probability theory, while the epistemic ones are treated with non-probabilistic approaches, e.g. evidence theory and possibility theory. In this paper, we only focus on aleatory (random) uncertainties and employ probability theory to deal with them. Therefore, based on the deterministic MDF-CSSO, the probabilistic version is also developed.

The rest of this paper is structured as follows. First, the deterministic MDF-CSSO procedure is developed, followed 
by the description of the probabilistic MDF-CSSO to realize uncertainty-based multidisciplinary design optimization. Second, one MDO benchmark test problem and one practical satellite conceptual design optimization problem are used to test the proposed methods and demonstrate the effectiveness. Finally, some conclusions are given, and future research is discussed.

\section{The MDF-CSSO procedure}

Consider a MDO problem with $N_{D}$ coupled disciplines as find $\mathbf{X}$

$\min f$

s.t. $\quad \mathbf{g} \leq 0$

$\mathbf{Y}_{i}=C A_{i}\left(\mathbf{X}_{i}, \mathbf{Y}_{\cdot i}\right) \quad i=1, \ldots N_{D}$

$\mathbf{X}=\underset{i=1, \ldots N_{D}}{\cup} \mathbf{X}_{i}, \mathbf{Y}=\underset{i=1, \ldots N_{D}}{\cup} \mathbf{Y}_{i}$,

$\mathbf{Y}_{\cdot i} \subseteq\left(\underset{j=1, \ldots N_{D}, j \neq i}{\cup} \mathbf{Y}_{j}\right)$

$\mathbf{X} \in \Omega, \quad f \in \mathbf{Y}, \mathbf{g} \subseteq \mathbf{Y}$

where $\mathbf{X}$ is the design variable vector with design space $\Omega$, $\mathbf{Y}$ is the state variable vector, $\mathbf{X}_{i}$ is the local design variable vector of discipline $i$ which is a sub-vector of $\mathbf{X}, \mathbf{Y}_{i}$ is the local output vector which is a sub-vector of $\mathbf{Y}$, and $\mathbf{Y}_{i}$ is the coupled state variable vector output from other disciplines and input into discipline $i$. There can be sharing of design variables between different disciplines, but $\mathbf{Y}_{i}$ are disjoint. The objective $f$ and the constraint vector $\mathbf{g}$ are sub-vectors of $\mathbf{Y} . C A_{i}$ is the contributing analysis (CA) of discipline $i$, which represents an analysis module contributing to the entire system analysis. A CA may be associated with a particular aspect of the system behavior or may represent a physical subsystem (Sobieszcznski-Sobieski 1988).

To solve (1), the proposed MDF-CSSO is sketched in Fig. 1. In the first stage, the disciplinary specialists are only responsible for building disciplinary surrogates, based on which system-level MDF is carried out to identify the optimum. For discipline $i$, the outputs are functions of the local design variables and the coupling input state variables from other disciplines, which are stated as $\mathbf{Y}_{i}=C A_{i}\left(\mathbf{X}_{i}, \mathbf{Y}_{\cdot i}\right)$. Accordingly, the surrogates are built as

$\tilde{\mathbf{Y}}_{i}=\tilde{\mathbf{Y}}_{i}\left(\mathbf{X}_{i}, \mathbf{Y}_{\cdot i}\right)=\tilde{\mathbf{Y}}_{i}\left(\mathbf{Q}_{i}\right)$

where $\mathbf{Q}_{i}=\left[\mathbf{X}_{i}, \mathbf{Y}_{. i}\right]$ is the local input variable vector of discipline $i$. It is worth noting that the surrogates (2) are different from the widely used surrogates $\tilde{\mathbf{Y}}_{i}(\mathbf{X})$ which are formulated as functions of the design variables $\mathbf{X}$. The selection of the surrogate formulation is based on the consideration for computational efficiency. Assume $N_{T}$ samples

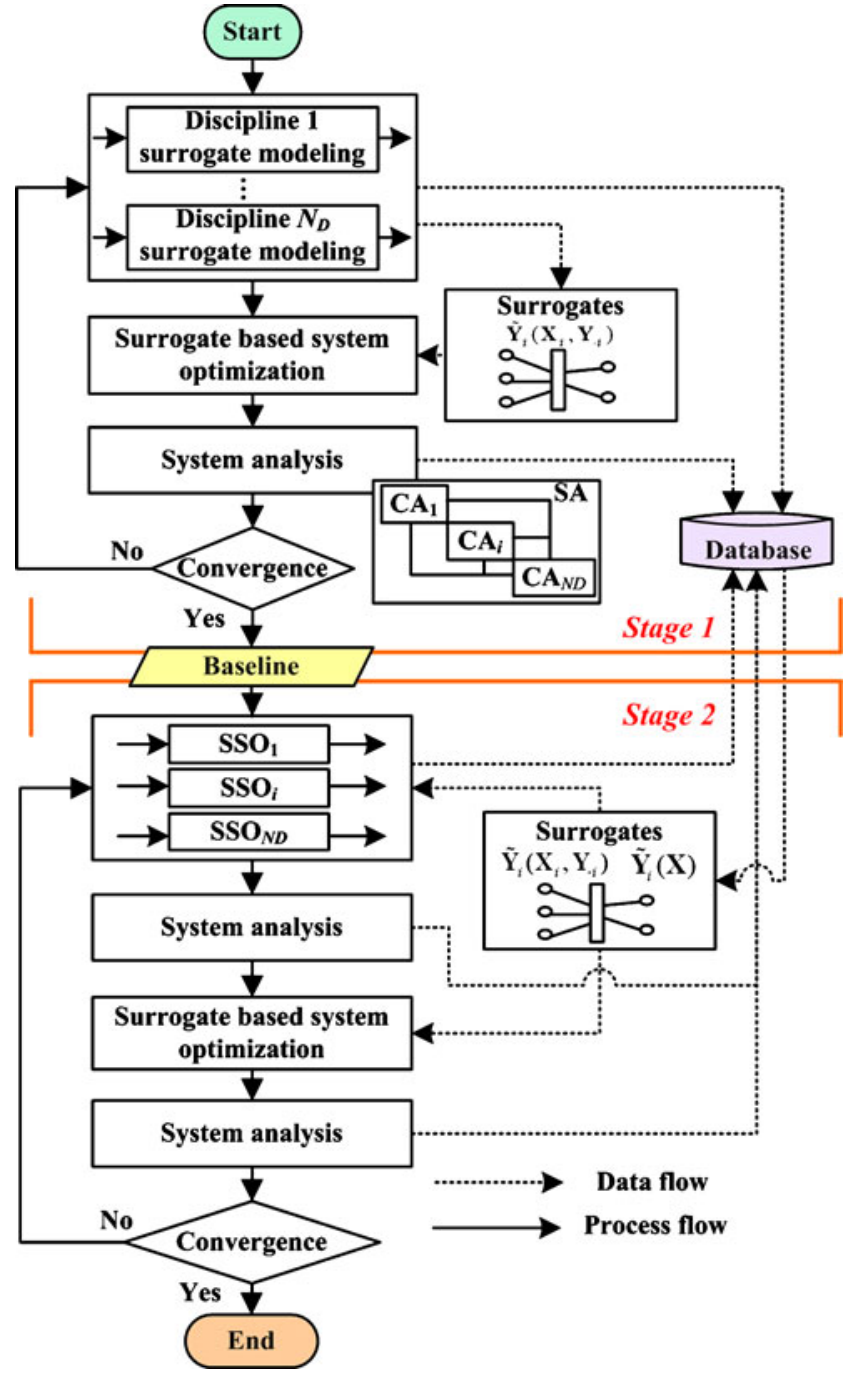

Fig. 1 Flowchart of MDF-CSSO

are needed to build $\tilde{\mathbf{Y}}_{i}(\mathbf{X})$. MDA is executed at each sample to obtain the corresponding output. Assume averagely $N_{C}$ iterations of disciplinary analyses are needed to reach a consistent result for each MDA. Then the total computational complexity to build all the disciplinary surrogates can be estimated as

$O\left(N_{C} \cdot N_{T} \cdot \sum_{i=1}^{N_{D}} O\left(C A_{i}\right)\right)$

where $O\left(C A_{i}\right)$ is the computational complexity of $C A_{i}$. Also assume $N_{T}$ samples are needed to build $\tilde{\mathbf{Y}}_{i}\left(\mathbf{X}_{i}, \mathbf{Y}_{i}\right)$. The total computational complexity is

$O\left(N_{T} \cdot \sum_{i=1}^{N_{D}} O\left(C A_{i}\right)\right)$ 
It is obvious that building $\tilde{\mathbf{Y}}_{i}\left(\mathbf{X}_{i}, \mathbf{Y}_{i}\right)$ is much cheaper. Moreover, the formulation $\tilde{\mathbf{Y}}_{i}\left(\mathbf{X}_{i}, \mathbf{Y}_{. i}\right)$ allows the disciplinary specialists to build the surrogates only with their own analysis tools and avoid coupling with other disciplinary analyses. Thus independent and concurrent operations can be realized to further improve efficiency. As the intermediate coupling variables $\mathbf{Y}_{. i}$ are directly used as the independent input in the surrogates, $\tilde{\mathbf{Y}}_{i}\left(\mathbf{X}_{i}, \mathbf{Y}_{. i}\right)$ are termed as intermediate surrogates in contrast to $\tilde{\mathbf{Y}}_{i}(\mathbf{X})$ which are only related to design variables.

As shown in the research of surrogate based optimization (Forrester and Keane 2009), it is desirable to accurately model the promising regions around the potential optimums, and it would be wasteful to obtain good accuracy all over the design space, especially for complex systems. An effective approach to address this issue is to run the surrogate based MDF sequentially, so that the surrogates can be initially built to be less accurate with less training cost and purposefully refined gradually with more information about promising regions obtained through optimization. The surrogate updating and MDF optimization are iteratively conducted until the convergence to the global optimum is attained. In this stage, the optimization formulation is the same as that of the original optimization problem, except that the highfidelity models are replaced with low-fidelity surrogates. Thus the global optimum of surrogate based MDF is equivalent to that of the original problem as long as the surrogates satisfy accuracy requirement in the promising region. Based on the optimum achieved in the first stage as baseline, the procedure proceeds to the second stage organized by CSSO. In this stage, the disciplinary specialists participate to investigate and improve the design through optimization with their high-fidelity analysis tools. To realize decomposition of the coupled disciplines so as to allow disciplinary autonomy, surrogates are used in each discipline to estimate non-local state variables. The system coordination is realized by system level optimization, wherein surrogates are also used to mitigate computational burden. This is essentially the same as the procedure of response surface based CSSO (CSSO-RS) (Sellar et al. 1996a). The algorithms of the two stages include the following steps.

Stage 1: $\quad$ Surrogate based MDF.

Stage 1.0: Initialization. Denote the cycle number $r=0$.

Stage 1.1: Disciplinary surrogate modeling. Denote the cycle number $r=r+1$.

Build surrogates $\tilde{\mathbf{Y}}_{i}^{(r)}\left(\mathbf{X}_{i}, \mathbf{Y}_{. i}\right)$ for each discipline. Training samples can be obtained with design of experiment (DOE) techniques. In this paper, the optimal Latin hypercube design (LHD) with maximin criterion is used to obtain uniformly scattered samples in the design space (Johnson et al. 1990). The domain of local design variables $\mathbf{X}_{i}$ can be directly defined by the design space specified in (1). The domain of coupling state variables can be firstly estimated roughly with larger ranges and refined with more information gained in later cycles. The optimums obtained in the previous cycles are also added into the training set to enhance the approximation accuracy in the potentially promising regions.

Stage 1.2: Surrogate based system optimization.

System optimization is carried out based on $\tilde{\mathbf{Y}}_{i}^{(r)}\left(\mathbf{X}_{i}, \mathbf{Y}_{\cdot i}\right)$, which is formulated as

find $\mathbf{X}^{(r)}$

$\min \tilde{f}$

s.t. $\quad \tilde{\mathbf{g}} \leq 0$

$\tilde{\mathbf{Y}}_{i}=\tilde{\mathbf{Y}}_{i}^{(r)}\left(\mathbf{X}_{i}^{(r)}, \tilde{\mathbf{Y}}_{\cdot i}\right) i=1, \ldots N_{D}$;

$\tilde{\mathbf{Y}}_{\cdot i} \subseteq\left(\underset{j=1, \ldots N_{D}, j \neq i}{\cup} \tilde{\mathbf{Y}}_{j}\right)$

$\mathbf{X}^{(r)} \in \Omega, \quad \tilde{f} \in \tilde{\mathbf{Y}}, \quad \tilde{\mathbf{g}} \subseteq \tilde{\mathbf{Y}}$

It can be noticed that the state variable surrogates are coupled in (5), which needs iterations to obtain a consistent output given a set of design variables. However, the cheap calculation cost of the surrogates makes it affordable. Denote the optimum obtained in previous cycles as $\mathbf{x}^{*}$, and use it as the baseline to solve (5) of current cycle. If $r=1$, the baseline is given arbitrarily.

Stage 1.3: System analysis.

The optimum $\mathbf{x}^{(r) *}$ of (5) in the $r$ th cycle is evaluated with accurate MDA, which is also called system analysis (SA). If $\mathbf{x}^{(r) *}$ is feasible (satisfying all the constraints) and better than the previous optimum, denote the optimum $\mathbf{x}^{*}=\mathbf{x}^{(r) *}$.

Stage 1.4: Check convergence.

The convergent criterion is that the average difference between the optimal solutions of three consecutive cycles should be smaller than a threshold $\varepsilon_{O}$ as

$$
\left(\left\|f^{(r) *}-f^{(r-1) *}\right\|+\left\|f^{(r-1) *}-f^{(r-2) *}\right\|\right) / 2 \leq \varepsilon_{O}
$$

If the convergent criterion is not satisfied, go back to step 1.1; otherwise, enter stage 2.

Stage 2: $\quad$ Surrogate based CSSO.

Stage 2.1: Denote the cycle number $r=r+1$. Define the optimum $\left(\mathbf{x}^{*}, \mathbf{y}^{*}\right)$ as the baseline $\left(\overline{\mathbf{x}}^{(r)}, \overline{\mathbf{y}}^{(r)}\right)$. Based on the existing samples and optimums 
obtained in previous cycles, build the surrogates $\tilde{\mathbf{Y}}_{i}^{(r)}(\mathbf{X})$ and $\tilde{\mathbf{Y}}_{i}^{(r)}\left(\mathbf{X}_{i}, \mathbf{Y}_{\cdot i}\right)$.

Stage 2.2: Concurrent subspace optimization (SSO). SSO $i$ is formulated as

$$
\begin{aligned}
& \text { find } \mathbf{X}_{i}^{(r)} \\
& \min \left\{\begin{array}{cc}
f, & f \in \mathbf{Y}_{i} \\
\tilde{f}, \quad f \notin \mathbf{Y}_{i}
\end{array}\right. \\
& \text { s.t. } \mathbf{g}_{i} \leq 0 \quad \mathbf{g}_{i} \subseteq \mathbf{Y}_{i} \\
& \mathbf{Y}_{i}=C A_{i}\left(\mathbf{X}_{i}^{(r)}, \mathbf{Y}_{\cdot i}\right) \\
& \forall \tilde{\mathbf{Y}}_{j} \subseteq \tilde{\mathbf{Y}}_{\cdot i}, \quad \tilde{\mathbf{Y}}_{j}=\tilde{\mathbf{Y}}_{j}^{(r)}\left(\mathbf{X}^{(r)}\right) \\
& \forall \mathbf{X}_{j}^{(r)} \subseteq \mathbf{X}^{(r)}(j \neq i), \quad \mathbf{X}_{j}^{(r)}=\overline{\mathbf{x}}_{j}^{(r)} \\
& \mathbf{X}_{i}^{(r)} \in \Omega_{i}
\end{aligned}
$$

where $\overline{\mathbf{x}}_{j}^{(r)}$ is the sub-vector of the baseline $\overline{\mathbf{x}}^{(r)}$ for the non-local design variables which are kept as constants in the $i$ th SSO. It is worth noting that herein the non-local state variables are calculated with $\tilde{\mathbf{Y}}_{j}(\mathbf{X})$ so as to avoid iteration with $C A_{i}$ to reach consistent analysis result for each design. The optimum of SSO $i$ is denoted as $\mathbf{x}_{i \_a l l}^{(r) *}=$ $\mathbf{x}_{i}^{(r) *} \cup \overline{\boldsymbol{x}}_{j=1 \ldots N_{D}, j \neq i}^{(r)}$. All the SSOs can be executed concurrently. During SSOs, the feasible design points visited during optimization are recorded and added into the training set to update $\tilde{\mathbf{Y}}_{i}^{(r)}\left(\mathbf{X}_{i}, \mathbf{Y}_{\cdot i}\right)$.

\section{Step 2.3: System analysis.}

The optimums obtained in SSOs are evaluated with accurate MDA. Denote the best feasible one as optimum $\mathbf{x}^{*}$.

Step 2.4: Surrogate based system optimization.

Step 2.5: System analysis.

Step 2.6: Check convergence.

The steps 2.4 to 2.6 are the same as the steps $1.2-1.4$. When the convergent criterion is reached, end the whole MDFCSSO procedure.

\section{The probabilistic MDF-CSSO procedure}

To account for uncertainties inevitably existing in engineering, MDF-CSSO is extended to solve uncertaintybased multidisciplinary design optimization (UMDO) problems, and the probabilistic version of MDF-CSSO (PMDFCSSO), is developed in this section.
The uncertainties considered in this paper include input uncertainties and model uncertainties. The input uncertainties are those associated with design variables. The model uncertainties include model structure uncertainties and model parameter uncertainties. Model structure uncertainties are mainly due to assumptions underlying the model which may not capture the physics correctly. They are problem dependent and not considered in this paper. Model parameter uncertainties are mainly due to limited information in estimating model parameters for a fixed model form (Batill et al. 2000; Du and Chen 2000a; de Weck et al. 2007), which can be universally dealt with by simply treating the system constants as uncertain ones. Both the uncertain design variables and the uncertain system parameters are assumed to be random and probability theory is used for uncertainty modeling and propagation.

Consider a UMDO problem with $N_{D}$ coupled disciplines as

find $\mu_{\mathbf{X}}$

$\min F\left(\mu_{f}, \sigma_{f}\right)$

s.t. $\operatorname{Pr}\{\mathbf{g} \leq 0\} \geq \mathbf{R}$

$\mathbf{Y}_{i}=C A_{i}\left(\mathbf{X}_{i}, \mathbf{Y}_{. i}, \mathbf{P}\right) \quad i=1, \ldots N_{D}$

$\mathbf{X}=\underset{i=1, \ldots N_{D}}{\cup} \mathbf{X}_{i}, \quad \mathbf{Y}=\underset{i=1, \ldots N_{D}}{\cup} \mathbf{Y}_{i}$,

$\mathbf{Y}_{\cdot i} \subseteq\left(\underset{j=1, \ldots N_{D}, j \neq i}{\cup} \mathbf{Y}_{j}\right)$

$\mu_{\mathbf{X}} \in \Omega, \quad f \in \mathbf{Y}, \quad \mathbf{g} \subseteq \mathbf{Y}$

where the design variable vector $\mathbf{X}$ and system parameter vector $\mathbf{P}$ are random, $\mu_{x}$ is the mean value of $\mathbf{X}$ to be optimized, $\mu_{f}$ and $\sigma_{f}$ are the mean and standard deviation of the objective $f, \operatorname{Pr}\{\cdot\}$ is the probability of the constraint within the braces to be satisfied, $\mathbf{R}$ is the reliability requirements for the constraints. Without loss of generality, the objective is formulated as the function of the mean and standard deviation of the original objective to incorporate robustness requirement for the objective. The robustness requirement for other state variables can also be considered as shown in Test 2 .

\subsection{PMDF-CSSO framework}

To solve (8), the proposed PMDF-CSSO framework is developed as follows.

Stage 1: Surrogate based MDF.

The algorithm of this stage is the same as that in MDFCSSO, except that the disciplinary surrogates are modeled 
as $\tilde{\mathbf{Y}}_{i}=\tilde{\mathbf{Y}}_{i}\left(\mathbf{X}_{i}, \mathbf{Y}_{\cdot i}, \mathbf{P}_{i}\right)$ where $\mathbf{P}_{i}$ is the local uncertain parameter vector, and the system level optimization problem under uncertainties in the $r$ th cycle is formulated as

$$
\begin{array}{ll}
\text { find } & \mu_{x}^{(r)} \\
\min \quad & F\left(\mu_{\tilde{f}}, \sigma_{\tilde{f}}\right) \\
\text { s.t. } \quad & \operatorname{Pr}\{\tilde{\mathbf{g}} \leq 0\} \geq \mathrm{R} \\
\tilde{\mathbf{Y}}_{i}= & \tilde{\mathbf{Y}}_{i}^{(r)}\left(\mathbf{X}_{i}^{(r)}, \tilde{\mathbf{Y}}_{\cdot i}, \mathbf{P}\right), \quad i=1, \ldots N_{D} ; \\
\tilde{\mathbf{Y}}_{i} \subseteq & \left(\underset{j=1, \ldots N_{D}, j \neq i}{\cup} \tilde{\mathbf{Y}}_{j}\right) \\
\mu_{x}^{(r)} \in \Omega, \quad \tilde{f} \in \tilde{\mathbf{Y}}, \quad \tilde{\mathbf{g}} \subseteq \tilde{\mathbf{Y}}
\end{array}
$$

\section{Stage 2: Surrogate based CSSO.}

The algorithm of this stage is the same as that in MDFCSSO, except that the surrogate models of non-local state variables in SSO is formulated as $\tilde{\mathbf{Y}}_{i}(\mathbf{X}, \mathbf{P})$, and SSO $i$ under uncertainties is

find $\mu_{\mathbf{X} i}^{(r)}$

$\min \begin{cases}F\left(\mu_{f}, \sigma_{f}\right), & f \in \mathbf{Y}_{i} \\ F\left(\mu_{\tilde{f}}, \sigma_{\tilde{f}}\right), & f \notin \mathbf{Y}_{i}\end{cases}$

s.t. $\operatorname{Pr}\left\{\mathbf{g}_{i} \leq 0\right\} \geq \mathbf{R}_{i}$

$\mathbf{Y}_{i}=C A_{i}\left(\mathbf{X}_{i}^{(r)}, \tilde{\mathbf{Y}}_{\cdot i}, \mathbf{P}_{i}\right)$

$\forall \tilde{\mathbf{Y}}_{j} \subseteq \tilde{\mathbf{Y}}_{\cdot i}, \quad \tilde{\mathbf{Y}}_{j}=\tilde{\mathbf{Y}}_{j}^{(r)}\left(\mathbf{X}^{(r)}\right)$

$\forall \mu_{\mathbf{X} j}^{(r)} \subseteq \mu_{\mathbf{X}}^{(r)}(j \neq i), \quad \mu_{\mathbf{X} j}^{(r)}=\mu_{\overline{\mathbf{X}}_{j}}^{(r)}, \quad \mu_{\mathbf{X} i}^{(r)} \in \Omega_{i}$

To solve the uncertainty-based optimization problems (9) and (10), the key is to characterize the uncertain features of system outputs resulting from the effects of input and model uncertainties by means of uncertainty analysis (Yao et al. 2010), which will be discussed in detail in next section.

\subsection{Uncertainty analysis}

Uncertainty analysis used to solve (9) and (10) includes two parts: estimation of the mean and standard deviation of the state variables to calculate the objective, and reliability analysis of the constraints. The moment estimation of state variables is solved by Taylor series approximation method in this paper for simplicity (Yao et al. 2011).
In (10), the non-local state variables are estimated by $\tilde{\mathbf{Y}}_{i}(\mathbf{X}, \mathbf{P})$, the mean and standard deviation of the vector element $\tilde{Y}_{i}^{j}$ can be estimated as

$\mu_{\tilde{Y}_{i}^{j}}=E\left(\tilde{Y}_{i}^{j}\right) \approx \tilde{Y}_{i}^{j}\left(\mu_{\mathbf{X}}, \mu_{\mathbf{P}}\right)$

$\sigma_{\tilde{Y}_{i}^{j}}^{2}=\sum_{k=1}^{n_{X}}\left(\frac{\partial \tilde{Y}_{i}^{j}}{\partial X^{k}}\right)^{2} \sigma_{X^{k}}^{2}+\sum_{k=1}^{n_{P}}\left(\frac{\partial \tilde{Y}_{i}^{j}}{\partial P^{k}}\right)^{2} \sigma_{P^{k}}^{2}$

where $n_{x}$ and $n_{p}$ are the numbers of design variables and model parameters, $\mu_{X}$ and $\mu_{P}$ are the mean values of $\mathbf{X}$ and $\mathbf{P}$, and $\sigma_{X^{k}}$ and $\sigma_{P^{k}}$ are the standard deviations of $X^{k}$ and $P^{k}$ respectively.

The local state variables are calculated with local accurate analysis models $C A_{i}$. The mean and standard deviation of the vector element $\tilde{Y}_{i}^{j}$ is estimated as

$$
\begin{aligned}
\mu_{Y_{i}^{j}}= & E\left(Y_{i}^{j}\right) \approx C A_{i}^{j}\left(\mu_{\mathbf{X}_{i}}, \mu_{\tilde{\mathbf{Y}}_{\cdot i}}, \mu_{\mathbf{P}_{i}}\right) \\
\sigma_{Y_{i}^{j}}^{2}= & \sum_{k=1}^{n_{X i}}\left(\frac{\partial Y_{i}^{j}}{\partial X_{i}^{k}}\right)^{2} \sigma_{X_{i}^{k}}^{2}+\sum_{k=1}^{n_{Y_{i}}}\left(\frac{\partial Y_{i}^{j}}{\partial \tilde{Y}_{\cdot i}^{k}}\right)^{2} \sigma_{\tilde{Y}_{\cdot i}^{k}}^{2} \\
& +\sum_{k=1}^{n_{P i}}\left(\frac{\partial Y_{i}^{j}}{\partial P_{i}^{k}}\right)^{2} \sigma_{P_{i}^{k}}^{2}
\end{aligned}
$$

Where $n_{X i}, n_{Y . i}$, and $n_{P i}$ are the numbers of local design variables, coupled input state variables, and local model parameters of discipline $i$ respectively, and $\sigma_{\tilde{Y}_{i}^{k}}^{2}$ are obtained from (12).

In (9), the state variables are estimated by intermediate surrogates which are coupled with each other. Hence the cross propagation of uncertainties should be considered. The mean and standard deviation are estimated as

$\mu_{\tilde{Y}_{i}^{j}}=E\left(\tilde{Y}_{i}^{j}\right) \approx \tilde{Y}_{i}^{j}\left(\mu_{\mathbf{X}_{i}}, \mu_{\tilde{\mathbf{Y}}_{. i}}, \mu_{\mathbf{P}_{i}}\right)$

$$
\begin{aligned}
\sigma_{\tilde{Y}_{i}^{j}}^{2}= & \sum_{k=1}^{n_{X i}}\left(\frac{\partial \tilde{Y}_{i}^{j}}{\partial X_{i}^{k}}\right)^{2} \sigma_{X_{i}^{k}}^{2}+\sum_{l=1 \ldots N_{D}, l \neq i}\left[\sum_{k=1}^{n_{Y l}}\left(\frac{\partial \tilde{Y}_{i}^{j}}{\partial \tilde{Y}_{l}^{k}}\right)^{2} \sigma_{\tilde{Y}_{l}^{k}}^{2}\right] \\
& +\sum_{k=1}^{n_{P i}}\left(\frac{\partial \tilde{Y}_{i}^{j}}{\partial P_{i}^{k}}\right)^{2} \sigma_{P_{i}^{k}}^{2}
\end{aligned}
$$

Denote $\sigma_{\tilde{\mathbf{Y}}_{i}}^{2}=\left[\sigma_{\tilde{Y}_{i}^{1}}^{2}, \ldots \sigma_{\tilde{Y}_{i}^{N_{Y i}}}^{2}\right]^{T}, \sigma_{\mathbf{X}_{i}}^{2}=\left[\sigma_{X_{i}^{1}}^{2}, \ldots \sigma_{X_{i}^{N_{X i}}}^{2}\right]^{T}$, $\sigma_{\mathbf{P}_{i}}^{2}=\left[\sigma_{P_{i}^{1}}^{2}, \ldots \sigma_{P_{i}^{N}{ }^{N P i}}^{2}\right]^{T}$, then

$\sigma_{\tilde{\mathbf{Y}}_{i}}^{2}=\mathbf{A}_{i} \cdot \sigma_{\mathbf{X}_{i}}^{2}+\sum_{j=1 \ldots N_{D}, j \neq i} \mathbf{B}_{i j} \cdot \sigma_{\tilde{\mathbf{Y}}_{j}}^{2}+\mathbf{C}_{i} \cdot \sigma_{\mathbf{P}_{i}}^{2}$ 
where

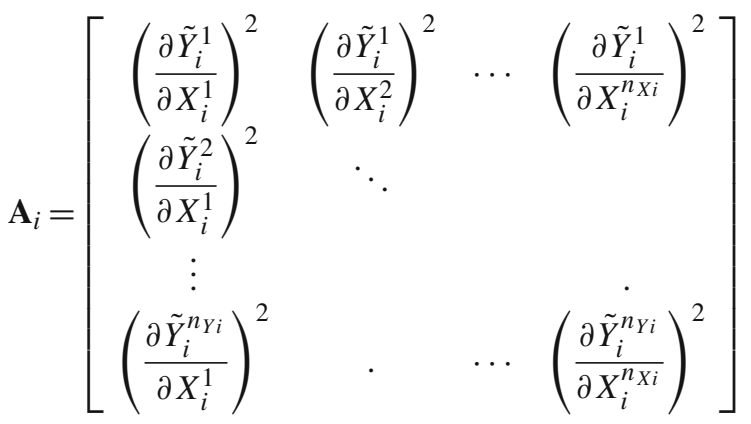

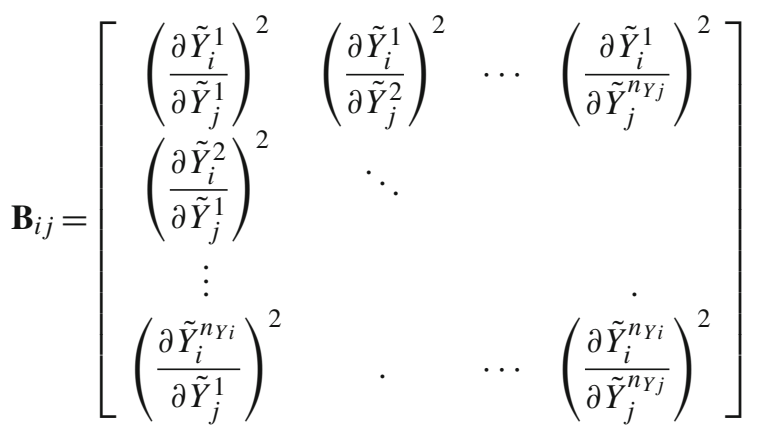

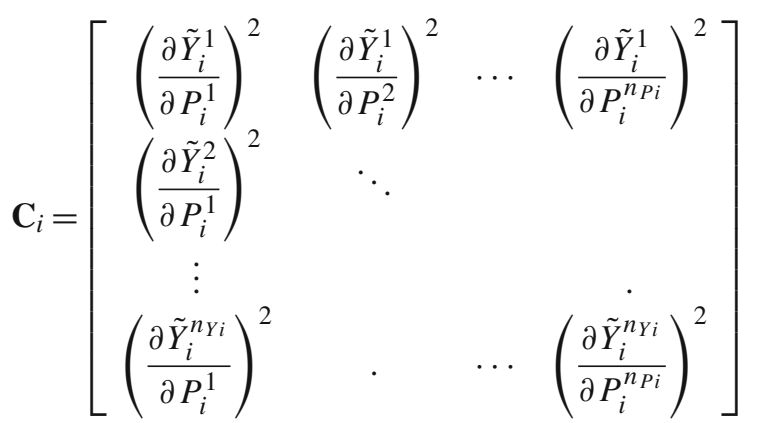

For all the SSOs, the equation system of (17) can be solved together as

$$
\left\{\begin{array}{c}
\cdot \\
\sigma_{\tilde{\mathbf{Y}}_{i}^{2}}^{2} \\
\sigma_{\tilde{\mathbf{Y}}_{i}}^{2}
\end{array}\right\}=\left[\begin{array}{ccccc}
I & & & & \cdot \\
& \cdot & & & \\
& & I & \cdot & -\mathbf{B}_{i j} \\
\cdot & \cdot & \cdot & \cdot & \cdot \\
\cdot & & -\mathbf{B}_{i j} & \cdot & I
\end{array}\right]^{-1}\left\{\begin{array}{c}
\cdot \\
\cdot \\
\mathbf{D}_{i} \\
\cdot \\
\mathbf{D}_{j} \\
\cdot
\end{array}\right\}
$$

$\mathbf{D}_{i}=\mathbf{A}_{i} \cdot \sigma_{\mathbf{X}_{i}}^{2}+\mathbf{C}_{i} \cdot \sigma_{\mathbf{P}_{i}}^{2}$

The preceding formulation of standard deviation estimation is essentially the same as that derived in ( $\mathrm{Du}$ and Chen 2000 b). Hence uncertainty analysis of the state variables can be realized analytically.

Compared to the moment estimation, reliability analysis is more complex especially for those with high reliability requirements, as small failure probability entails intensive computation to calculate. Since reliability of constraints should be quantified at every search point during optimization, the design space search process is nested with reliability analysis which is so called double-loop optimization. Thus it is computationally prohibitive, especially in subspace optimization of stage 2 wherein accurate disciplinary models are used. There are several approaches in the literature to address the coupled reliability-based optimization problems, e.g. single level approaches (SLA) that either merge the inner reliability analysis loop and outer optimization loop into one single level problem (Chen et al. 1997; Agarwal et al. 2004; Liang and Mourelatos 2008) or decouple the double loop into sequential cycles of reliability analysis and deterministic MDO (Royset et al. 2001; Wu et al. 2001; Du and Chen 2002), which are surveyed in (Valdebenito and Schuëller 2010). Herein the SLA method proposed in (Chen et al. 1997) is used for simplicity. In optimization, the reliability constraint $\operatorname{Pr}\{\mathrm{g}(\mathbf{Q}) \leq 0\}$ $\geq R_{g}$ is reformulated as

$G\left(\mathbf{z}^{(k)}\right) \leq 0$

$\mathbf{z}^{(k)}=\mu_{\mathbf{z}}^{(k)}+\beta \alpha^{*(k-1)}$

$\mu_{\mathbf{z}}^{(k)}=\mathbf{Q} / \sigma_{\mathbf{Q}} ; \quad \alpha^{*(k-1)}=\nabla_{\mathbf{z}} G\left(\mathbf{z}^{(k-1)}\right) /\left\|\nabla_{\mathbf{z}} G\left(\mathbf{z}^{(k-1)}\right)\right\|$

where the limit state function $G$ is the counterpart of constraint $g$ in the uncorrelated normalized space, $\mathbf{z}^{(k)}$ is the approximate MPP (Most Probable Point) of input $\mathbf{Q}$ in the $k$ th iteration of optimization, $\alpha^{*(k-1)}$ is the vector of direction cosine of the constraint at the MPP $\mathbf{z}^{(k-1)}$ of previous iteration, $\beta$ is the reliability index corresponding to the reliability requirement $R_{g}$. The main idea of this formulation is to check whether the reliability constraint is satisfied by comparing the percentile value with required reliability against the limit state value, which can save a lot of computation as accurate reliability does not need to be calculated. The problem is the percentile value is calculated at the approximate MPP which is estimated based on the direction cosine at the MPP of the previous iteration which may be inaccurate. However, after several iterations, $\mathbf{z}$ can converge to the accurate MPP and the optimal reliable design can be obtained.

As (23) dose not calculate the real constraint reliability, an additional reliability analysis, e.g. MCS, First Order Reliability Method (FORM) or Second Order Reliability Method (SORM), etc., is needed after optimization to calculate the real reliability. This can be carried out in the step of system analysis, where the optimums obtained in the system or subspace optimization are analyzed with accurate MDA. In this paper, MCS is used for its accuracy and ease of implementation. Besides, the rich samples generated during MCS can be reused to update surrogate models. 


\section{Tests}

\subsection{Test 1}

The MDF-CSSO procedure is firstly testified with the speed reducer optimization problem, which is one of the benchmark problems for MDO test (Padula et al. 1996). The problem is stated as

find : $\mathbf{X}=\left[\begin{array}{lllllll}x_{1} & x_{2} & x_{3} & x_{4} & x_{5} & x_{6} & x_{7}\end{array}\right]^{T}$

$\min : f(\mathbf{X})=0.7854 x_{1} x_{2}^{2}$

$$
\begin{aligned}
& \times\left(3.3333 x_{3}^{2}+14.9334 x_{3}-43.0934\right) \\
& -1.5079 x_{1}\left(x_{6}^{2}+x_{7}^{2}\right)+7.477\left(\mathrm{x}_{6}^{3}+x_{7}^{3}\right) \\
& +0.7854\left(x_{4} x_{6}^{2}+x_{5} x_{7}^{2}\right)
\end{aligned}
$$

s.t. $g_{1}: 27.0 /\left(x_{1} x_{2}^{2} x_{3}\right)-1 \leq 0$,

$$
\begin{aligned}
& g_{2}: 397.5 /\left(x_{1} x_{2}^{2} x_{3}^{2}\right)-1 \leq 0 \\
& g_{3}: 1.93 x_{4}^{3} /\left(x_{2} x_{3} x_{6}^{4}\right)-1 \leq 0, \\
& g_{4}: 1.93 x_{5}^{3} /\left(x_{2} x_{3} x_{7}^{4}\right)-1 \leq 0 \\
& g_{5}: A_{1} / B_{1}-1100 \leq 0, \\
& g_{6}: A_{2} / B_{2}-850 \leq 0 \\
& g_{7}: x_{2} x_{3}-40.0 \leq 0 \text {, } \\
& g_{8}: 5.0 \leq x_{1} / x_{2} \\
& g_{9}: x_{1} / x_{2} \leq 12.0, \\
& g_{10}:\left(1.5 x_{6}+1.9\right) / x_{4}-1 \leq 0 \\
& g_{11}:\left(1.1 x_{7}+1.9\right) / x_{5}-1 \leq 0 \\
& A_{1}=\left[\left(\frac{745.0 x_{4}}{x_{2} x_{3}}\right)^{2}+16.9 \times 10^{6}\right]^{0.5}, B_{1}=0.1 x_{6}^{3} \\
& A_{2}=\left[\left(\frac{745.0 x_{5}}{x_{2} x_{3}}\right)^{2}+157.5 \times 10^{6}\right]^{0.5}, B_{2}=0.1 x_{7}^{3} \\
& 2.6 \leq x_{1} \leq 3.6,0.7 \leq x_{2} \leq 0.8,17 \leq x_{3} \leq 28, \\
& 7.3 \leq x_{4} \leq 8.3 \\
& 7.3 \leq x_{5} \leq 8.3,2.9 \leq x_{6} \leq 3.9,5.0 \leq x_{7} \leq 5.5
\end{aligned}
$$

With reference to (Tosserams et al. 2007), the problem is decomposed into three disciplines (subsystems). Discipline 1 is concerned with gear design, while disciplines 2 and 3 are responsible for the design of two shafts. The disciplinary settings are defined as follows.

Discipline $1: \mathbf{X}_{1}=\left[\begin{array}{lll}x_{1} & x_{2} & x_{3}\end{array}\right]^{T}$,

$$
\begin{aligned}
& \mathbf{Y}_{1}=\left[\begin{array}{lllllll}
g_{1} & g_{2} & g_{7} & g_{8} & g_{9} & f & y_{1}
\end{array}\right]^{T}, \\
& \mathbf{Y}_{\cdot 1}=\left[\begin{array}{ll}
y_{2} & y_{3}
\end{array}\right]^{T}
\end{aligned}
$$

Discipline 2: $\mathbf{X}_{2}=\left[\begin{array}{lll}x_{1} & x_{4} & x_{6}\end{array}\right]^{T}, \mathbf{Y}_{2}=\left[\begin{array}{llll}g_{3} & g_{5} & g_{10} & y_{2}\end{array}\right]^{T}$,

$$
\mathbf{Y}_{\cdot 2}=\left[y_{1}\right]^{T}
$$

Discipline $3: \mathbf{X}_{3}=\left[\begin{array}{lll}x_{1} & x_{5} & x_{7}\end{array}\right]^{T}, \mathbf{Y}_{3}=\left[\begin{array}{llll}g_{4} & g_{6} & g_{11} & y_{3}\end{array}\right]^{T}$,

$$
\mathbf{Y}_{3}=\left[y_{1}\right]^{T}
$$

where

$$
\begin{aligned}
f= & 0.7854 x_{1} x_{2}^{2}\left(3.3333 x_{3}^{2}+14.9334 x_{3}-43.0934\right) \\
& +y_{2}+y_{3} \\
y_{1}= & x_{2} \cdot x_{3} \\
y_{2}= & -1.5079 x_{1} x_{6}^{2}+7.477 x_{6}^{3}+0.7854 x_{4} x_{6}^{2} \\
y_{3}= & -1.5079 x_{1} x_{7}^{2}+7.477 x_{7}^{3}+0.7854 x_{5} x_{7}^{2}
\end{aligned}
$$

The optimization problem is solved with MDF-CSSO. In stage one, the surrogates of the eleven constraints $g_{1}$ to $g_{11}$, the three coupling state variables $y_{1}$ to $y_{3}$, and the objective $f$, are modeled. For example, the surrogate of the objective is modeled as $\tilde{f}=\tilde{f}\left(x_{1}, x_{2}, x_{3}, y_{2}, y_{3}\right)$, and the constraint $g_{3}$ in disciplinary 2 is modeled as $\tilde{g}_{3}=\tilde{g}_{3}\left(x_{4}, x_{6}, y_{1}\right)$. In surrogate modeling of each discipline, 60 samples uniformly distributed in the domain of local input are used to train the surrogates. The domain of design variables are directly defined in (24). The domain of coupling state variables are defined by solving (25). Based on the preceding surrogates, MDF is implemented by formulating the optimization problem in the form of (5). Sequential quadratic programming (SQP) is employed as optimization solver. Each time the optimum is obtained, it is added into the training data to rebuild the surrogates, so that the accuracy of the surrogates in the promising region can be gradually improved. With the updated surrogates, optimization (5) is resolved to identify new optimum. After iterations of preceding two steps, the convergence to the optimum can be achieved with surrogates satisfying predefined accuracy requirements in the promising region. 


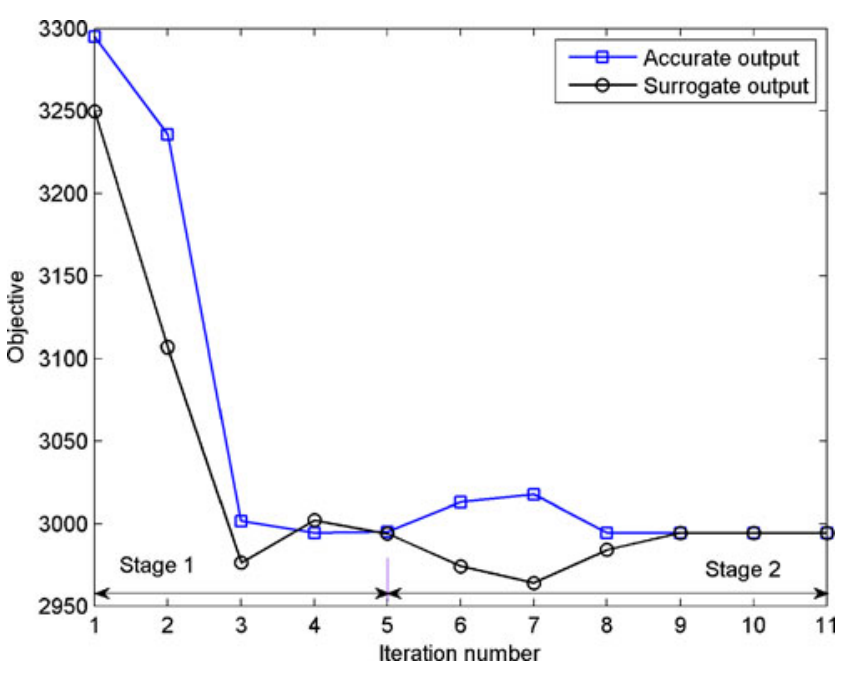

Fig. 2 Convergence history of objective in Test 1

a

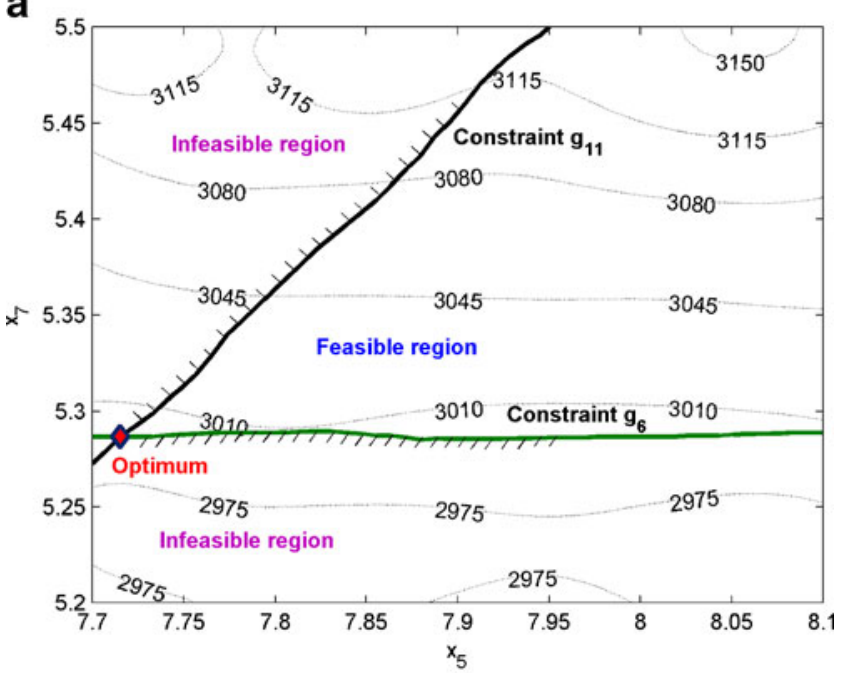

b

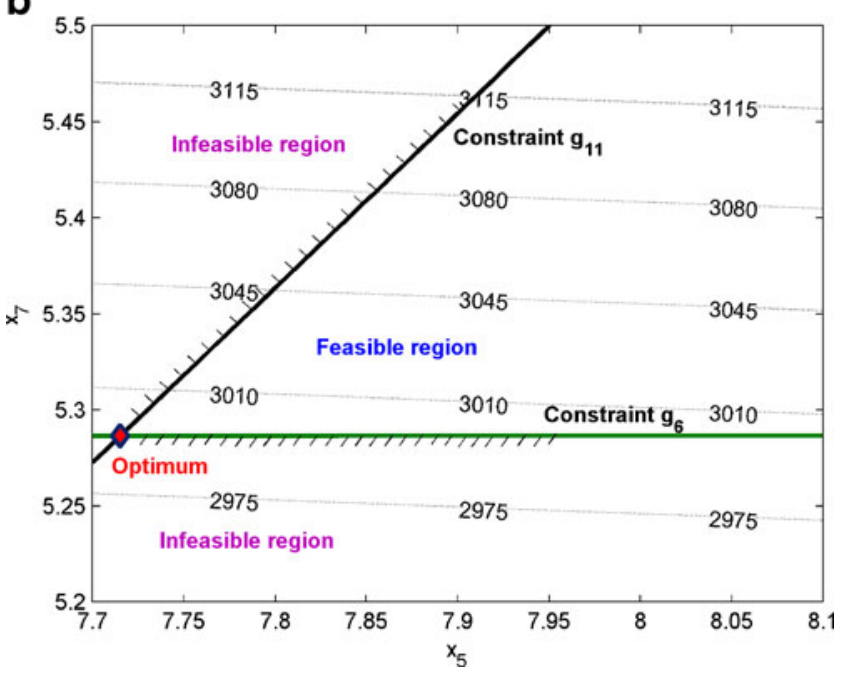

Fig. 3 a The surrogate and $\mathbf{b}$ the accurate models of the objective and the active constraints with respect to $x_{5}$ and $x_{7}$ at the optimum where $x_{1}=3.5, x_{2}=0.7, x_{3}=17, x_{4}=7.3$, and $x_{6}=3.3502$

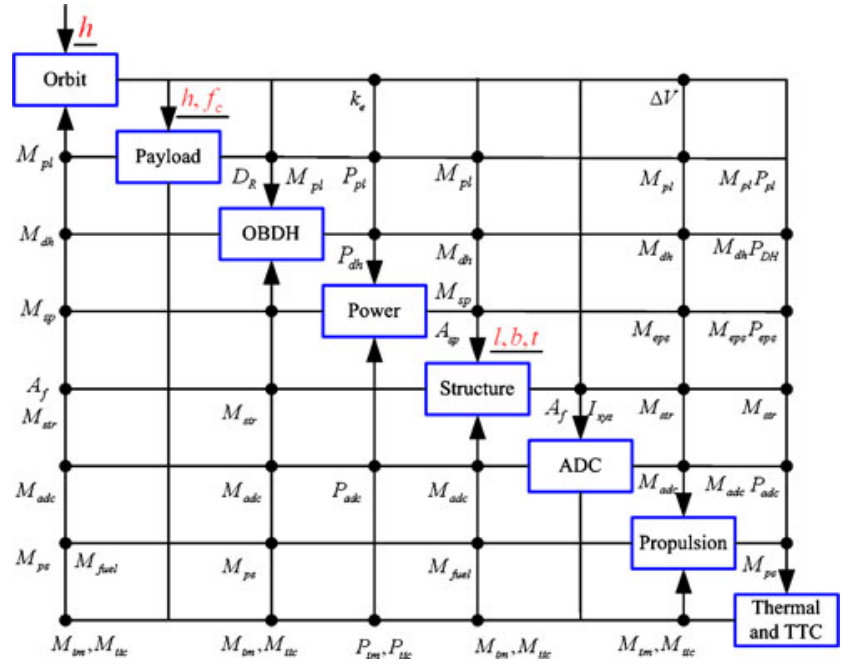

Fig. 4 Design structure matrix of satellite system design in Test 2

Taking the optimum achieved in the first stage as baseline, CSSO is further implemented to refine the optimal design with concurrent subspace optimizations. Firstly, the

Table 1 Nomenclature in Test 2

\begin{tabular}{ll}
\hline Symbol & Description \\
\hline$h$ & Orbit altitude \\
$k_{e}$ & Eclipse factor \\
$b$ & Body width \\
$D_{R}$ & Data rate of payload \\
$F_{s t r}$ & Structure safety index \\
$M_{e p s} P_{e p s}$ & Mass and power of power subsystem \\
$M_{p l} P_{p l}$ & Mass and power of payload \\
$M_{a d c} P_{a d c}$ & Mass and power of ADC \\
$M_{t t c} P_{t t c}$ & Mass and power of TTC \\
$f_{c}$ & Focal length \\
$\Delta V$ & Delta V budget \\
$l$ & Body height \\
$M_{s t r}$ & Mass of structure \\
$V_{s a t}$ & Satellite volume \\
$A_{s p}$ & Area of solar panels \\
$M_{d h} P_{d h}$ & Mass and power of OBDH \\
$M_{p}$ & Mass of propulsion \\
$M_{t m} P_{t m}$ & Mass and power of thermal subsystem \\
$R$ & Resolution \\
$S_{W}$ & Coverage swath \\
$t$ & Side wall thickness \\
$I_{x}, I_{y} I_{z}$ & Moments of inertia \\
$M_{s a t}$ & Satellite mass \\
$M_{s p}$ & Mass of solar panels \\
$A_{f}$ & Area of front face \\
$M_{f u e l}$ & \\
\hline &
\end{tabular}


Table 2 Uncertain design variables in Test 2

\begin{tabular}{llll}
\hline Discipline & Variables & $\begin{array}{l}\text { Distribution } \\
\text { type }\end{array}$ & $\begin{array}{l}\text { Distribution } \\
\text { parameters }\end{array}$ \\
\hline Structure & Width $/ \mathrm{mm}$ & Normal & std 0.5 \\
& Height $/ \mathrm{mm}$ & Normal & std 0.5 \\
& Thickness $/ \mathrm{mm}$ & Normal & std 0.01 \\
Orbit & Altitude $/ \mathrm{km}$ & Normal & std 0.5 \\
Payload & Focal length $/ \mathrm{mm}$ & Normal & std 0.1 \\
\hline
\end{tabular}

surrogates of state variables with respect to design variables are modeled based on the samples obtained in the first stage. For example, the surrogate of the objective is modeled as $\tilde{f}=\tilde{f}\left(x_{1}, x_{2}, x_{3}, x_{4}, x_{5}, x_{6}, x_{7}\right)$. The SSO is formulated in the form of (4), and SQP is also used as solver. In each SSO, the local state variables are directly calculated with the accurate models, e.g. the constraints $g_{1}$ and $g_{2}$ in discipline 1 are directly calculated with the equations stated in (24). If non-local state variables are needed as input for the local accurate models, the surrogates of these non-local state variables are used for estimation. When all the SSOs are solved, the optimums are evaluated with system analysis and the best one is chosen as the baseline for system optimization which is formulated in the form of (5). Herein the surrogates used in (5) are enhanced with data points obtained during SSOs which have been analyzed with local highfidelity models. Then the optimum of (5) is passed down to all the SSOs for further optimization. After several iterations of subspace optimization and system optimization, the convergence to the final optimum can be achieved.

In this test, as no noise is involved, all the surrogates are built with interpolation RBF neural network (RBFNN) models for its advantage in approximating highly nonlinear functions (Park and Sandberg 1991; Jin et al. 2000).

All the optimizations from four different initial points stated in (Zhao and Cui 2011) stably converge to the optimum [3.5. 0.7, 17, 7.3, 7.7153, 3.3502, 5.2867] with the objective $f=2994.355$. One of the convergence histories of the objective is depicted in Fig. 2. In stage 1, it only takes five iterative cycles to quickly converge to the solution which is close to the real optimum. Based on this baseline, six more iterative cycles in stage 2 are taken to further refine the design, which converge to the real optimum by employing concurrent SSOs with accurate disciplinary analysis tools. Due to the efficiency of MDF which can provide a good baseline for CSSO, the drawback of CSSO as too many iterative cycles are needed to converge can be circumvented. The contour plots of the objective with respect to $x_{5}$ and $x_{7}$ at the optimum are illustrated in Fig. 3. It shows that the surrogates of the objective and the active constraints agree well with the accurate models in the region around the optimum, which is the premise of surrogate based optimization to converge to the global optimum of the original MDO problem.

\subsection{Test 2}

The efficacy of the proposed PMDF-CSSO procedure is demonstrated with a hypothetical earth observatory small satellite design problem (Yao et al. 2010).

\section{- Satellite Design Modeling}

The mission is to observe a specific area with minimum resolution of $30 \mathrm{~m}$ and minimum coverage swath of $50 \mathrm{~km}$. The objective is to minimize the satellite mass. The disciplines involved in the design include orbit, payload, structure,
Table 3 Uncertain model parameters in Test 2

\begin{tabular}{llll}
\hline Discipline & Parameters & Distribution type & Distribution parameters \\
\hline Structure & Launch vehicle axial natural frequency $/ \mathrm{Hz}$ & Normal & Mean 30.0 std 0.3 \\
& Launch vehicle lateral natural frequency $/ \mathrm{Hz}$ & Normal & Mean 15.0 std 0.15 \\
& Axial overload coefficient & Normal & Mean 6.0 std 0.06 \\
& Lateral overload coefficient & Normal & Mean 3.0 std 0.03 \\
& Axial ultimate tensile strength $/ \mathrm{N} / \mathrm{m}^{2}$ & Log normal & Mean 4.2e8 std 4.2e5 \\
& Axial stretch yield stress / N/m ${ }^{2}$ & Log normal & Mean 3.2e8 std 3.2e5 \\
& Young's modulus / N/m ${ }^{2}$ & Normal & Mean 7.1e10 std 7.1e7 \\
Thermal & Power estimation scaling factor & Normal & Mean 0.04 std 0.0004 \\
& Mass estimation scaling factor & Interval & {$[0.045,0.055]$} \\
TTC & Power estimation scaling factor & Normal & Mean 0.045 std 0.00045 \\
& Mass estimation scaling factor & Interval & {$[0.045,0.055]$} \\
OBDH & Power estimation scaling factor & Normal & Mean 0.05 std 0.0005 \\
& Mass estimation scaling factor & Interval & {$[0.04,0.05]$} \\
\hline
\end{tabular}


and other subsystems, e.g. onboard data handling (OBDH), power, attitude determination and control (ADC), propulsion, thermal, and telemetry, tracking and command (TTC). The orbit is a sun synchronous circular orbit. Eccentricity is zero and inclination is fixed with given orbit altitude. The values of true anomaly, argument of perigee, and right ascension of ascending node are only related to ground track and irrelevant to the satellite mass and observing resolution estimation. Therefore, these parameters are not considered in optimization and only orbit altitude $h$ is taken as a design variable. The payload is a CCD camera with working spectrum from 0.4 to $0.9 \mu \mathrm{m}$. The design variable is focal length $f_{c}$, based on which the payload mass and power are predicted with empirical scaling equations (Yao 2007). The satellite configuration is simplified as a cube, and the cross section perpendicular to the flying direction is assumed to be square. Thus the design variables only include width $b$, height $l$, and side wall thickness $t$. The structure strength, stiffness and stability safeties under launch conditions are checked with empirical equations, and the parameter settings are taken from the Chinese launch vehicle CZ4B. According to the design of orbit, payload, and structure, the sizing of other subsystems can be estimated with empirical relationships for conceptual design (Wertz and Larson 1999).

The coupling relationships of the disciplines are described with a design structure matrix shown in Fig. 4. The symbols in the diagram are explained in Table 1 . The underlined symbols represent aforementioned design variables.

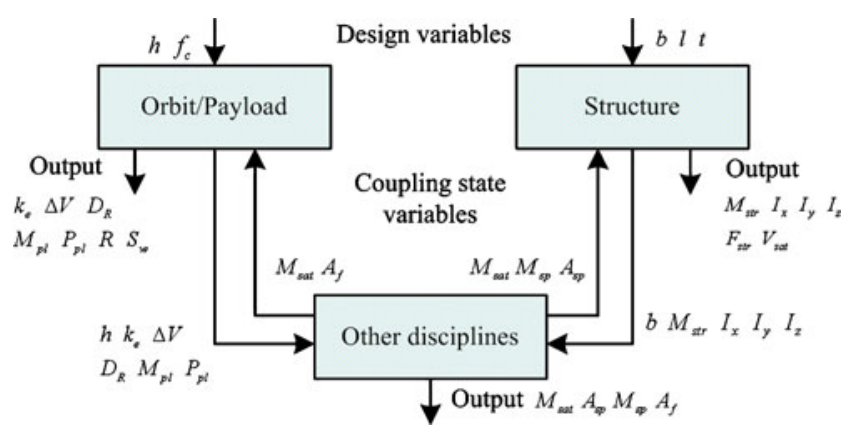

Fig. 5 The coupling relationship between the three decomposed subspaces of the satellite UMDO in Test 2

Given a set of design variable values, the satellite sizing can be estimated.

\section{- Uncertainty modeling}

The five design variables are assumed to be uncertain with normal distribution. The uncertainties with structure sizes and the optical lens are primarily induced by manufacturing tolerance. The orbit altitude uncertainty mainly results from orbit perturbation. The mean values are to be optimized, and the standard deviations are fixed and listed in Table 2. The uncertain model parameters include the structure material uncertainties (e.g. Young's modulus, material density, etc.), the launch vehicle uncertainties (e.g. the launch vehi-

Table 4 Optimization results of MDF, PMDF, MDF-CSSO, and PMDF-CSSO in Test2

\begin{tabular}{|c|c|c|c|c|c|c|c|c|c|c|}
\hline & Variables & Baseline & MDF & & PMDF & & MDF-CS & SO & PMDF- & SSO \\
\hline \multirow[t]{5}{*}{ Design variables } & $h / \mathrm{km}$ & 650 & 597.15 & & 596.55 & & 607.46 & & 607.23 & \\
\hline & $f_{c} / \mathrm{mm}$ & 250 & 278.67 & & 281.36 & & 285.48 & & 286.48 & \\
\hline & $b / \mathrm{mm}$ & 750 & 851.45 & & 852.95 & & 851.85 & & 852.14 & \\
\hline & $l / \mathrm{mm}$ & 750 & 706.18 & & 706.58 & & 708.00 & & 708.25 & \\
\hline & $t / \mathrm{mm}$ & 7.5 & 5.00 & & 5.00 & & 5.00 & & 5.00 & \\
\hline \multirow[t]{2}{*}{ Active constraints } & $R / \mathrm{m}$ & & 30.00 & $\operatorname{Pr}=0.52$ & 29.68 & $\operatorname{Pr}=1$ & 29.93 & $\operatorname{Pr}=0.69$ & 29.67 & $\operatorname{Pr}=1$ \\
\hline & $V_{\text {sat }} / \mathrm{m}^{3}$ & & 0.50 & $\operatorname{Pr}=0.48$ & 0.502 & $\operatorname{Pr}=1$ & 0.501 & $\operatorname{Pr}=0.81$ & 0.502 & $\operatorname{Pr}=1$ \\
\hline \multirow[t]{4}{*}{ Objectives } & $\mu_{M_{s a t}} / \mathrm{kg}$ & & 180.69 & & 181.39 & & 181.42 & & 181.71 & \\
\hline & $\sigma_{M_{s a t}} / \mathrm{kg}$ & & 1.78 & & 1.65 & & 1.80 & & 1.69 & \\
\hline & $\sigma_{R} / \mathrm{m}$ & & 0.056 & & 0.055 & & 0.061 & & 0.059 & \\
\hline & $f$ & & 1.488 & & 1.457 & & 1.510 & & 1.479 & \\
\hline \multirow[t]{4}{*}{ Other characteristics } & \multicolumn{2}{|c|}{ Cycle number ${ }^{\mathrm{a}}$} & 53 & & 12 & & 32 & & 9 & \\
\hline & \multicolumn{2}{|c|}{ Total MDA calls } & 319 & & 12,073 & & 32 & & 9,009 & \\
\hline & \multicolumn{2}{|c|}{ Total CA calls } & 5,231 & & 197,974 & & 4,250 & & 166,450 & \\
\hline & \multicolumn{2}{|c|}{ Computational time /s } & 3,828 & & 144,876 & & 3,110 & & 121,807 & \\
\hline
\end{tabular}

${ }^{a}$ For MDF and PMDF, the cycle number is the number of design space search steps. For MDF-CSSO and PMDF-CSSO, the cycle number is the number of iterative cycles which cover a complete loop of surrogate-based MDF or CSSO from surrogate modeling to optimization 


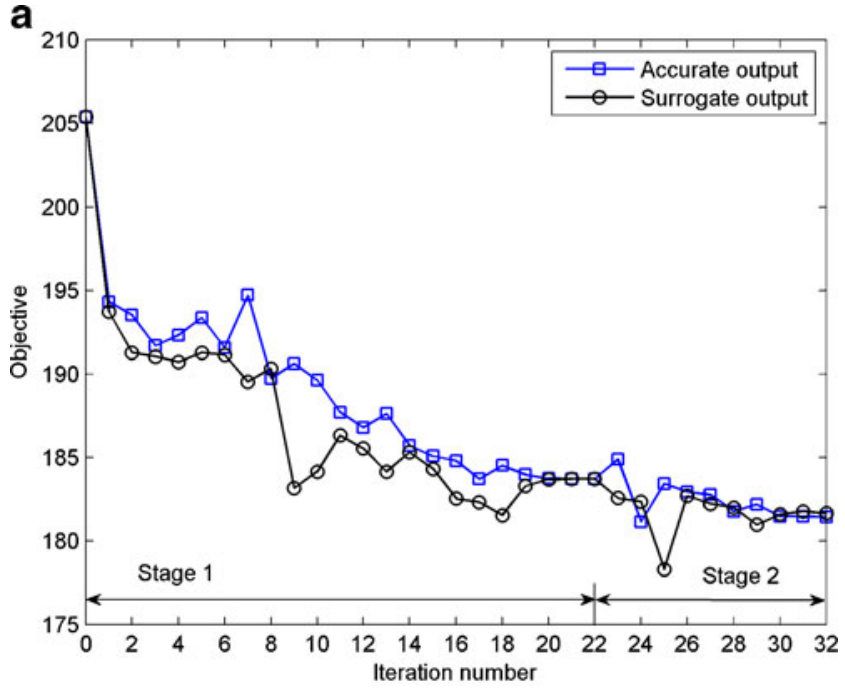

b

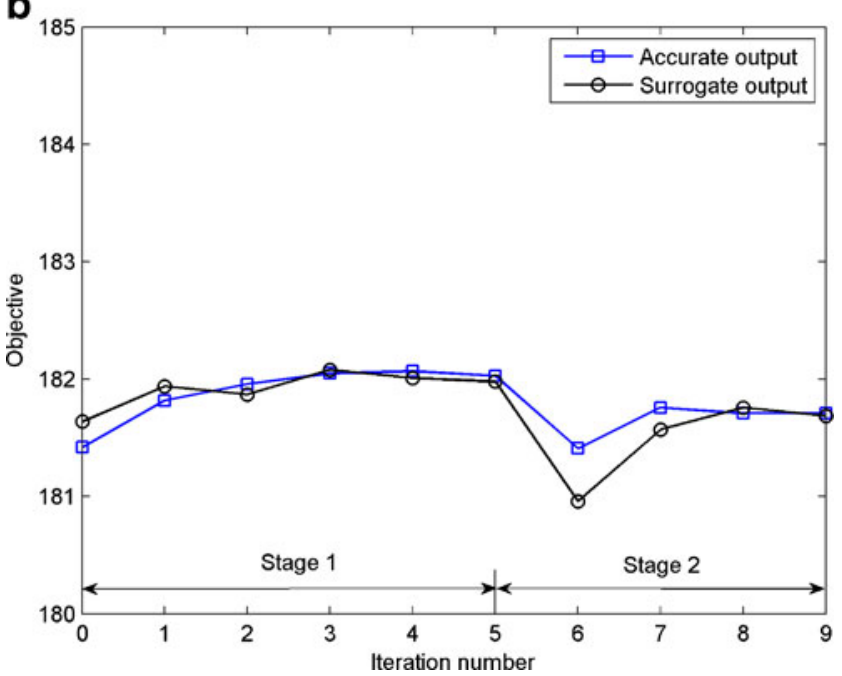

Fig. 6 Convergence history of a MDF-CSSO and b PMDF-CSSO in Test 2

cle axial natural frequency, axial overload coefficient, etc.), and the uncertainties associated with the simplified empirical equations in satellite sizing (e.g. the scaling parameters of the mass estimation models). As it would be computationally prohibitive to take all the uncertainties into account, sensitivity analysis is used to find out the factors with significant influences. The analysis shows thirteen uncertain system parameters should be considered, which are listed in Table 3 .

\section{- UMDO problem formulation}

There are three optimization objectives. One is to minimize the mean value of satellite mass $\left(\mu_{M_{s a t}}\right)$, which is directly related to cost. The second is to minimize the standard deviation of satellite mass $\left(\sigma_{M_{\text {sat }}}\right)$, which is related to cost risk. The third is to minimize the standard deviation of resolution $\left(\sigma_{R}\right)$, so as to maintain robustness of observation performance under uncertain effects. Therefore, the optimization is a multi-objective problem. In this paper, these three objectives are linearly summed into a single-objective for simplification.

Five constraints are set to embody design requirements. The satellite volume $V_{\text {sat }}$ should be no less than $0.5 \mathrm{~m}^{3}$ to accommodate instrument installation. The structure safety index (the ratio between the design thickness and the critical thickness of failure) $F_{s t r}$ should be no less than 1 . The observation resolution $R$ should be no larger than $30 \mathrm{~m}$. The coverage swath $S_{w}$ should be no less than $50 \mathrm{~km}$. The orbit eclipse factor $k_{e}$ should be no larger than 0.35 for charging requirement (Yao 2007). All the constraints are required to be satisfied under uncertainties with probability being no less than 0.9999 .

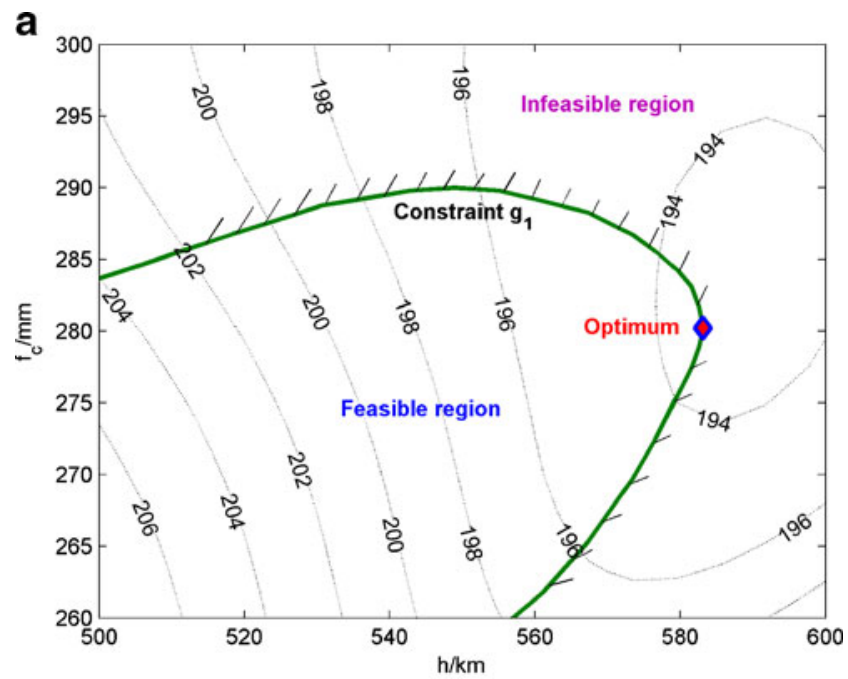

b

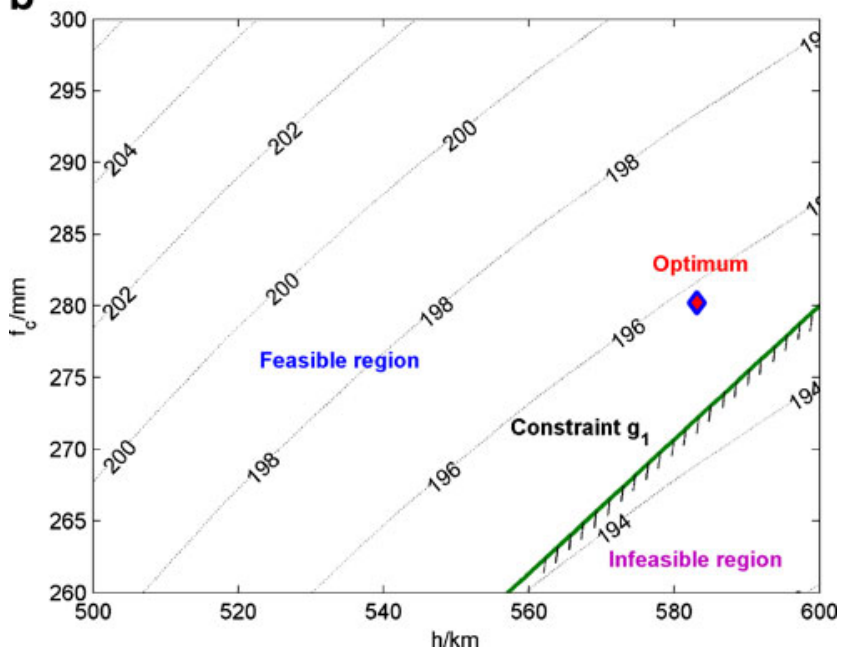

Fig. 7 Contour plots of the objective and active constraints at the optimum of the first cycle of MDF-CSSO in Test 2. a The surrogate and b the accurate models of the objective and the active constraints with respect to $h$ and $f_{c}$, where $b=913.34, l=744.06$, and $t=5$ 
To sum up, the UMDO problem is formulated as

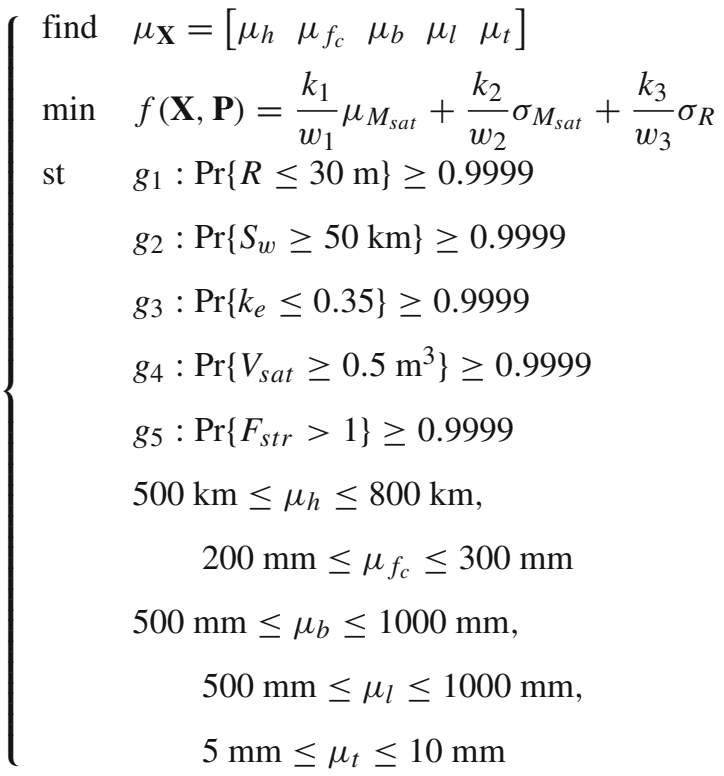

where $w_{i}$ are scalar factors to adjust the three objectives to be of the same order, and $k_{i}$ are weight coefficients to adjust preference, which are set as $k_{1}=0.5, k_{2}=k_{3}=0.25, w_{1}=$ $100, w_{2}=1$, and $w_{3}=0.1$.

According to the coupling degree between disciplines and distribution of design variables, the UMDO problem is decomposed into three subspace problems. One is the integration of orbit and payload which are closely coupled to define the observation capability. The second is structure. The third is the combination of all the other subsystems of satellite bus which have no design variables. The coupling relationship is described in Fig. 5, and the disciplinary settings are as follows.

Subspace $1: \mathbf{X}_{1}=\left[\begin{array}{ll}h & f_{c}\end{array}\right]^{T}$,

$$
\begin{aligned}
& \mathbf{Y}_{1}=\left[\begin{array}{lllllll}
k_{e} & \Delta V & D_{R} & M_{p l} & P_{p l} & R & S_{w}
\end{array}\right]^{T} \\
& \mathbf{Y}_{\cdot 1}=\left[\begin{array}{ll}
M_{\text {sat }} & A_{f}
\end{array}\right]^{T}, \mathbf{G}_{1}=\left[\begin{array}{lll}
g_{1} & g_{2} & g_{3}
\end{array}\right]^{T}
\end{aligned}
$$

Subspace $2: \mathbf{X}_{2}=\left[\begin{array}{lll}b & l & t\end{array}\right]^{T}$,

$$
\begin{aligned}
\mathbf{Y}_{2} & =\left[\begin{array}{llllll}
M_{s t r} & I_{x} & I_{y} & I_{z} & F_{\text {str }} & V_{\text {sat }}
\end{array}\right]^{T} \\
\mathbf{Y}_{\cdot 2} & =\left[\begin{array}{lll}
M_{s a t} & M_{s p} & A_{s p}
\end{array}\right]^{T}, \mathbf{G}_{2}=\left[\begin{array}{ll}
g_{4} & g_{5}
\end{array}\right]^{T}
\end{aligned}
$$

Subspace $3: \mathbf{X}_{3}=[], \mathbf{Y}_{3}=\left[\begin{array}{llll}M_{\text {sat }} & A_{s p} & M_{s p} & A_{f}\end{array}\right]^{T}$

$$
\begin{aligned}
& \mathbf{Y}_{\cdot 3}=\left[\begin{array}{llllllllll}
h & k_{e} & \Delta V & D_{R} & M_{p l} & b & M_{\text {str }} & I_{x} & I_{y} & I_{z}
\end{array}\right]^{T} \text {, } \\
& \mathbf{G}_{3}=[]
\end{aligned}
$$

- Results and discussion

This UMDO problem is firstly solved with MDF-CSSO to identify the deterministic optimum, based on which
PMDF-CSSO is implemented to satisfy the robustness and reliability requirements under uncertainties. The interpolation RBFNN models are used to build the surrogates, and the sample sizes to train the RBFNN of discipline 1, 2, and 3 are 80,120 , and 200, respectively. The optimization solvers of SSO and system optimization are SQP. This UMDO problem is also solved with the benchmark method MDF based on accurate models for comparison. To accommodate uncertainties, the same uncertainty analysis method stated in PMDF-CSSO is integrated in MDF, termed as PMDF. MDF is firstly conducted to identify deterministic optimum, based on which PMDF is further executed to locate the optimum under uncertainties. The baselines of MDF-CSSO and MDF are both the median point of the design space. In the system analysis of PMDF and PMDF-CSSO, MCS

a

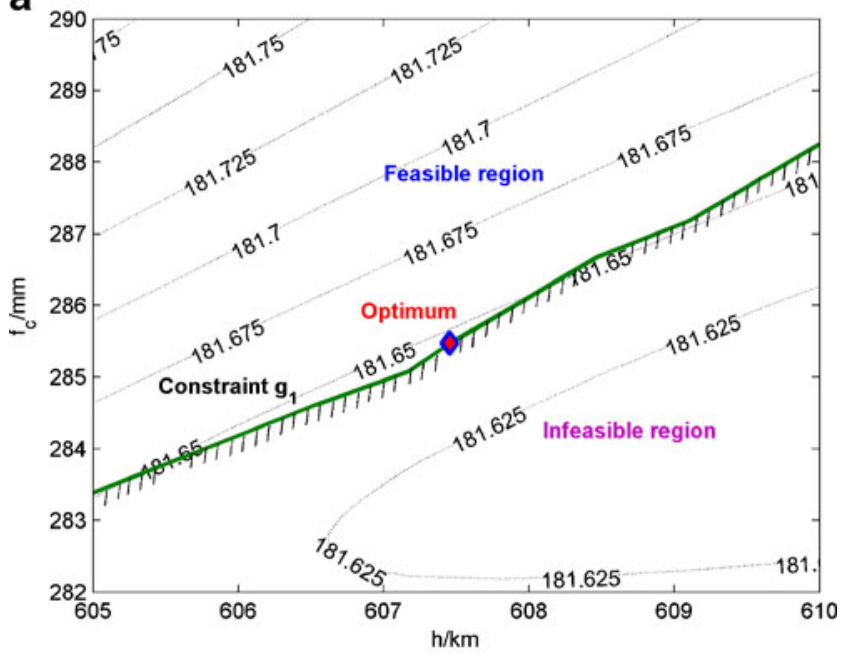

b

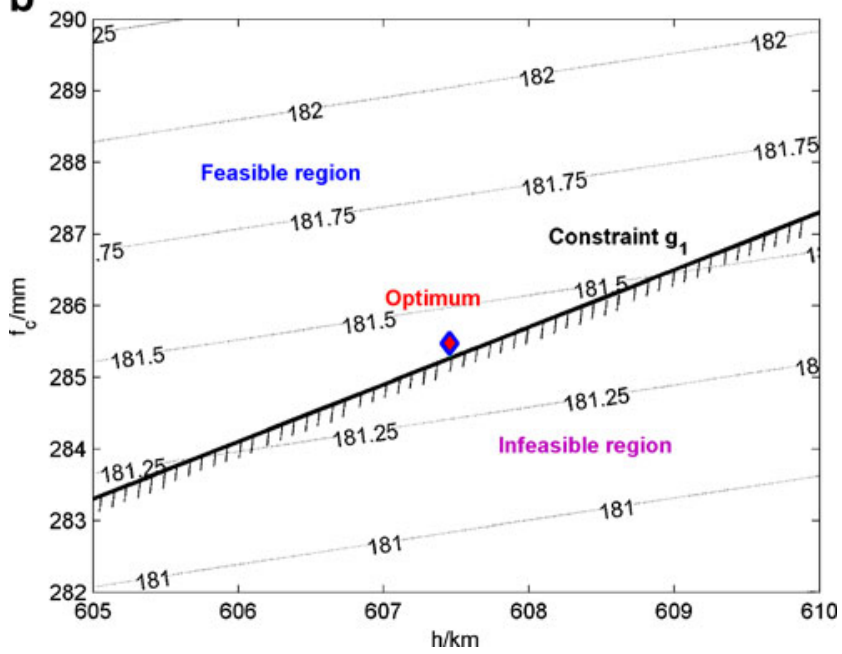

Fig. 8 Contour plots of the objective with active constraints at the optimum of the last cycle of MDF-CSSO in Test 2. a The surrogate and $\mathbf{b}$ the accurate models of the objective and the active constraints with respect to $h$ and $f_{c}$, where $b=851.85, l=708.00$, and $t=5$ 
with sample size of 1,000 is used to calculate the accurate constraint reliability.

The optimization results are listed in Table 4. It shows that the optimums of the proposed methods are very close to those obtained by MDF and PMDF, and the relative difference is less than $0.5 \%$. Besides, MDF-CSSO and PMDF-CSSO cost much less CA calls and computing time than MDF and PMDF by $15.5 \%$ and $15.9 \%$ respectively, which greatly enhance the optimization efficiency. The optimums of MDF and MDF-CSSO are located on the active constraints $g_{1}$ and $g_{4}$, hence the failure probabilities of these two constraints are much higher than the desired level. Based on the deterministic optimums, both PMDF and PMDF-CSSO entail no more than 20 cycles to locate the

a

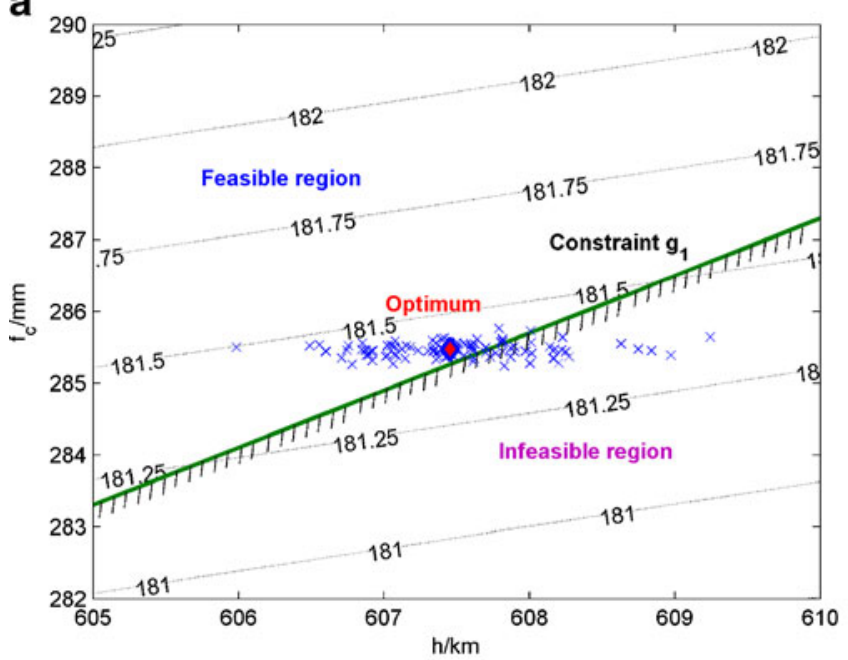

C

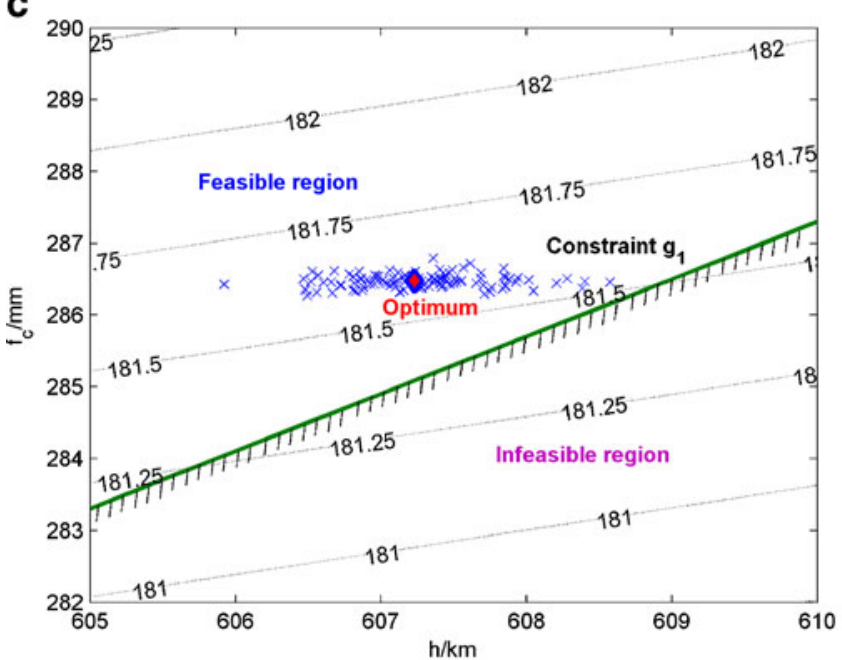

Fig. 9 MCS scatter plots at the optimums of MDF-CSSO and PMDFCSSO with accurate models in Test 2. a The objective and the active constraints with respect to $h$ and $f_{c}$ at the optimum of MDF-CSSO, where $b=851.85, l=708.00$, and $t=5$. b The objective and the active constraints with respect to $b$ and $l$ at the optimum of MDFCSSO, where $h=607.46, f_{c}=285.48$, and $t=5$. c The objective optimum under uncertainties. The reliability requirements of the active constraints are satisfied and the robustness of resolution is improved. It can be noticed that the computation of PMDF and PMDF-CSSO is dramatically higher than that of deterministic MDF and MDF-CSSO, which demonstrates the computational complexity of UMDO. However, it is worthwhile as the reliability and robustness can be enhanced.

The convergence history of MDF-CSSO and PMDFCSSO are depicted in Fig. 6, and the contour plots of the objective at the optimums of the first and last cycle of MDFCSSO are portrayed in Figs. 7 and 8, respectively. It can be observed that the surrogates in the first cycle are very inaccurate, but after several cycles the accuracy is greatly
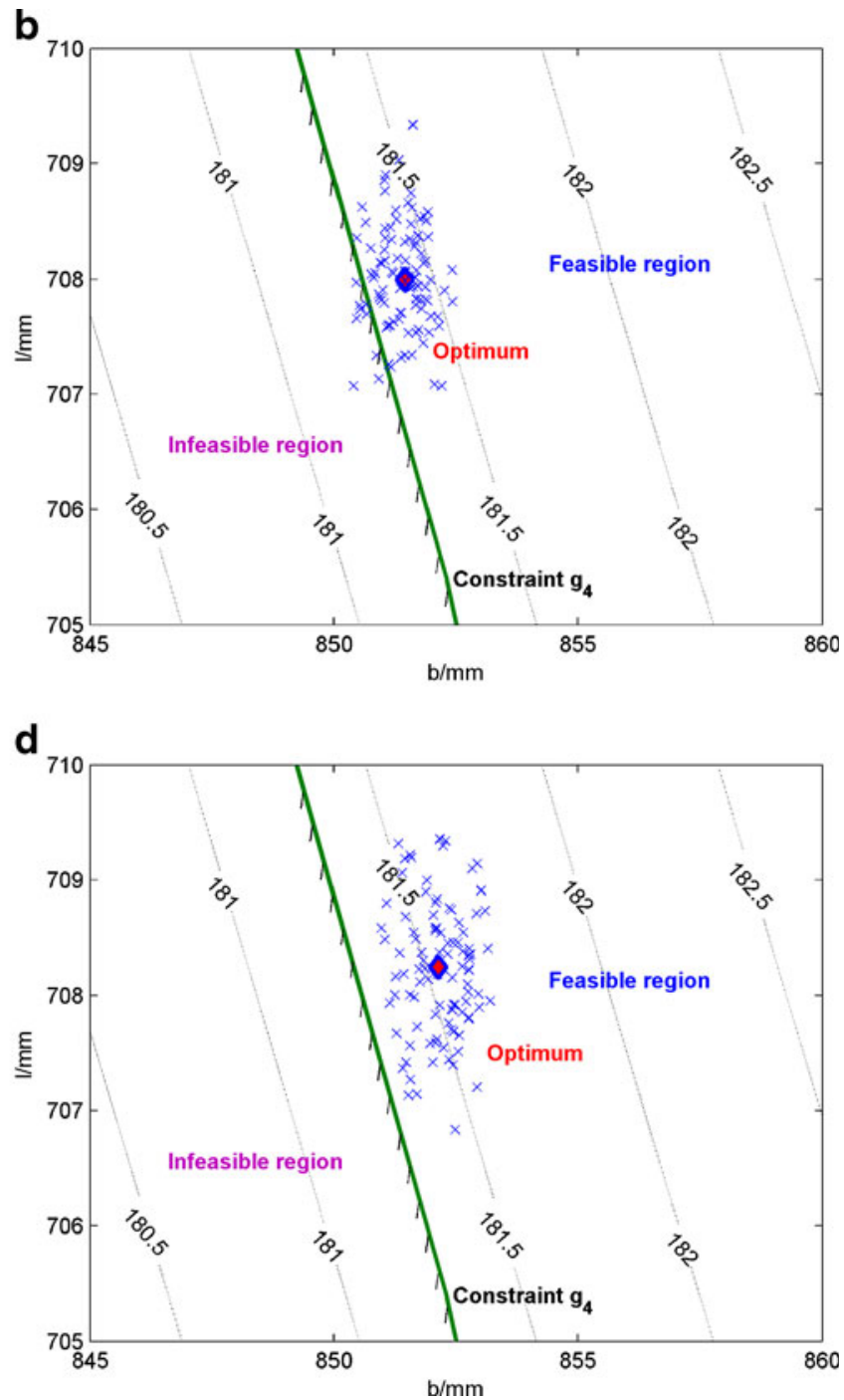

and the active constraints with respect to $h$ and $f_{c}$ at the optimum of PMDF-CSSO, where $b=852.14, l=708.25$, and $t=5$. d The objective and the active constraints with respect to $b$ and $l$ at the optimum of PMDF-CSSO, where $h=607.23, f_{c}=286.48$, and $t=5$ 
improved in the promising region. In Fig. 9, the MCS scatter plots at the optimums of MDF-CSSO and PMDF-CSSO are presented respectively. It clearly shows how the slight shift from the optimum of MDF-CSSO to the optimum of PMDF-CSSO enhances the reliability under uncertainty.

\section{Conclusions}

In this paper, a multistage-multilevel MDO procedure is proposed by integrating MDF and CSSO. The main idea is to organize MDO elements by complying with the realistic procedure of satellite system design and optimization in conceptual design phase. In the first stage, the system level specialists are involved to generate and assess alternative designs, and quickly identify promising solutions with low-fidelity models. To mimic this process, MDF is used to quickly locate the optimum of the MDO problem based on the approximation surrogates of disciplinary models which are built by disciplinary specialists independently. In the second stage, the disciplinary specialists are employed to investigate and improve the baseline obtained in the first stage with high-fidelity disciplinary models. To allow autonomy of disciplinary design and optimization, decomposition based procedure CSSO is used to organize concurrent disciplinary optimization and system coordination. Surrogates are used in subspace optimization to estimate non-local state variables so as to enable decomposition. This MDF-CSSO procedure can not only provide a framework to organize MDO following the realistic engineering conventions, but also improve the efficiency by exploiting merits and circumventing drawbacks of each single optimization procedure. To sum up, MDF-CSSO has the following advantages. First, the stability and efficiency of MDF can guarantee the convergence to the promising region. As surrogates are used, the computational cost of MDF becomes affordable. Second, the difficulty of surrogate-based MDF, that lengthy process is needed to adjust the surrogates accurately and converge to the real optimum, can be solved as CSSO is used to investigate the promising region by means of concurrent disciplinary optimization with high-fidelity disciplinary models. Third, based on the optimum of MDF as the baseline, the convergent difficulty of CSSO can be alleviated. Further considering that there exist uncertainties in practical engineering, the probabilistic MDF-CSSO (PMDF-CSSO) is also developed to solve uncertainty-based multidisciplinary design optimization problems. The effectiveness of the proposed methods is testified with one MDO benchmark test and one practical satellite conceptual design optimization problem. The results show that MDF-CSSO and PMDF-CSSO can quickly converge to the designs close to the real optimums of the original optimization problems with much less computational cost compared to MDF with accurate models, which demonstrate the effectiveness and efficiency of the proposed methods.

The major challenge of the proposed methods lies in the surrogate modeling. The optimization efficiency is significantly influenced by the surrogate accuracy. If the initial surrogates are too rough, it may fail to characterize the landscape of the underlying model, and the optimization could be trapped in the fake local optimum of the surrogates. However, it would be too expensive to build surrogates which are accurate all over the design space, as modeling the promising regions accurately is enough. Hence, the strategy of surrogate modeling and surrogate based optimization needs further investigation in the future research.

Acknowledgments This work was supported in part by National Natural Science Foundation of China under Grant No. 50975280 and Grant No. 61004094, Program for New Century Excellent Talents in University of Ministry of Education of China under Grant No. NCET-08-0149, Fund of Innovation by Graduate School of National University of Defense Technology under Grant No. B090102, and Hunan provincial innovation foundation for postgraduate, China.

\section{References}

Agarwal H, Renaud J, Lee J, Watson L (2004) A unilevel method for reliability based design optimization. In: Proceedings of the 45th AIAA/ASME/ASCE/AHS structures, structural dynamics, and materials conference, Palm Springs. AIAA-2004-2029

Alexandrov NM, Kodiyalam S (1998) Initial results of an mdo method evaluation study. In: 7th AIAA/USAF/NASA/ISSMO symposium on multidisciplinary analysis and optimization, St. Louis, Missouri. AIAA-1998-4884

Balling RJ, Sobieszcznski-Sobieski J (1996) Optimization of coupled systems: a critical overview of approach. AIAA J 34:6-17

Balling RJ, Wilkinson CA (1997) Execution of multidisciplinary design optimization approaches on common test problems. AIAA J 35:178-186

Batill SM, Renaud JE, Gu X (2000) Modeling and simulation uncertainty in multidisciplinary design optimization. In: 8th AIAA/USAF/NASA/ISSMO symposium on multidisciplinary analysis and optimization, Long Beach, CA. AIAA-2000-4803

Chen X, Hasselman TK, Neill DJ (1997) Reliability based structural design optimization for practical applications. In: Proceedings of the 38th AIAA/ASME/ASCE/AHS structures, structural dynamics, and materials conference, Kissimmee. AIAA-1997-1403

Chen S, Zhang F, Khalid M (2002) Evaluation of three decomposition mdo algorithms. In: Proceedings of 23rd international congress of aerospace sciences, Toronto, Canada

DeMiguel V, Murray W (2006) A local convergence analysis of bilevel decomposition algorithms. Optim Eng 7:99-133

de Weck O, Eckert C, Clarkson J (2007) A classification of uncertainty for early product and system design. In: International conference on engineering design, Paris, France. ICED07/No.480

Du X, Chen W (2000a) A methodology for managing the effect of uncertainty in simulation-based design. AIAA J 38:14711478

Du X, Chen W (2000b) An efficient approach to probabilistic uncertainty analysis in simulation-based multidisciplinary design. In: 
the 38th AIAA aerospace sciences meeting and exhibit, Reno, Nevada. AIAA-2000-0423

Du X, Chen W (2002) Sequential optimization and reliability assessment method for efficient probabilistic design. In: Proceedings of ASME 2002 design engineering technical conference and computers and information in engineering conference, Montreal. DETC2002/DAC-34127

Forrester AIJ, Keane AJ (2009) Recent advances in surrogate-based optimization. Prog Aerosp Sci 45:50-79

Helton JC, Burmaster DE (1996) Treatment of aleatory and epistemic uncertainty in performance assessments for complex systems. Reliab Eng Syst Saf 54:91-94

Hoffman FO, Hammonds JS (1994) Propagation of uncertainty in risk assessments: the need to distinguish between uncertainty due to lack of knowledge and uncertainty due to variability. Risk Analysis 14:707-712

Jin R, Chen W, Simpson TW (2000) Comparative studies of metamodeling techniques under multiple modeling criteria. AIAA-20004801

Johnson M, Moore L, Ylvisaker D (1990) Minimax and maximin distance designs. J Stat Plan Infer 26:131-148

Liang J, Mourelatos ZP (2008) A single-loop method for reliabilitybased design optimisation. Int J Prod Dev 5:76-92

Luo W (2003) Study and application of theory of multimethod collaborative optimization in the system design of flying vehicles. $\mathrm{PhD}$ Thesis, National University of Defense Technology

Padula SL, Alexandrov N, Green LL (1996) MDO test suite at NASA Langley research center. In: 6th AIAA/NASA/ISSMO symposium on multidisciplinary analysis and optimization. AIAA-1996-4028

Park J, Sandberg IW (1991) Universal approximation using radial basis function networks. Neural Comput 3:246-257

Royset JO, Der Kiureghian A, Polak E (2001) Reliability-based optimal structural design by the decoupling approach. Reliab Eng Syst Saf 73:213-221

Sellar RS, Batill SM (1996) Concurrent subspace optimization using gradient-enhanced neural network approximations. In: 6th AIAA, NASA, and ISSMO symposium on multidisciplinary analysis and optimization, Reston, VA. AIAA-96-4019

Sellar RS, Batill SM, Renaud JE (1996a) Response surface based, concurrent subspace optimization for multidisciplinary system design. In: 34th AIAA aerospace sciences meeting and exhibit, Reno, NV. AIAA-96-0714

Sellar RS, Stelmack MA, Batill SM, Rnaud JE (1996b) Response surface approximations for discipline coordination in multidis- ciplinary design optimization. In: 37th AIAA/ASME/AHS/ASC Structures, Structural Dynamics and Materials Conference, Salt Lake City, Utah. AIAA-96-2311

Simpson TW, Booker AJ, Ghosh D, Giunta AA, Koch PN, Yang R (2004) Approximation methods in multidisciplinary analysis and optimization: a panel discussion. Struct Optim 27:302-313

Sobieszcznski-Sobieski J (1988) Optimization by decomposition: a step from hierarchic to non-hierarchic systems. In: Second NASA/Air Force symposium on recent advances in multidisciplinary analysis and optimization, Hampton, Virginia

Sobieszczanski-Sobieski J, Haftka RT (1997) Multidisciplinary aerospace design optimization survey of recent developments. Struct Optim 14:1-23

Tedford NP, Martins JRRA (2010) Benchmarking multidisciplinary design optimization algorithms. Optim Eng 11:159-183. doi:10.1007/s11081-009-9082-6

Tosserams S, Etman LFP, Rooda JE (2007) An augmented Lagrangian decomposition method for quasi-separable problems in mdo. Struct Multidisc Optim 34:211-227

Valdebenito M, Schuëller G (2010) A survey on approaches for reliability-based optimization. Struct Multidisc Optim 42:645663

Wertz JR, Larson WJ (1999) Space mission analysis and design. 3rd ed. Microcosm Press, California

Wu YT, Shin Y, Sues R, Cesare M (2001) Safety-factor based approach for probability-based design optimization. In: 42nd AIAA/ASME/ASCE/AHS/ASC structures, structural dynamics and materials conference and exhibit, Seattle, Washington. AIAA-2001-1522

Yao W (2007) Research on uncertainty multidisciplinary design optimization theory and application to satellite system design. Master Thesis, National University of Defense Technology

Yao W, Guo J, Chen X, van Tooren M (2010) Utilizing uncertainty multidisciplinary design optimization for conceptual design of space systems. In: 8th Conference on systems engineering research, Hoboken, NJ, USA

Yao W, Chen X, Luo W, van Tooren M, Guo J (2011) Review of uncertainty-based multidisciplinary design optimization methods for aerospace vehicles. Prog Aerosp Sci. 47(6):450-479. doi: 10.1016/j.paerosci.2011.05.001

Yi SI, Shin JK, Park GJ (2008) Comparison of MDO methods with mathematical examples. Struct Multidisc Optim 35:391-402

Zhao M, Cui W (2011) On the development of bi-level integrated system collaborative optimization. Struct Multidisc Optim 43:73-84 